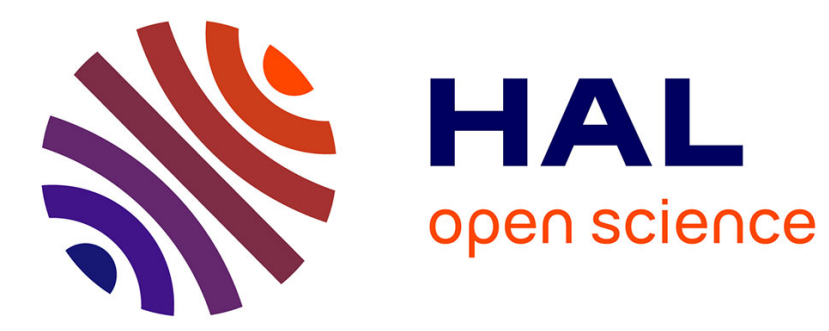

\title{
Patterns in Wall-Bounded Shear Flows
}

Laurette S. Tuckerman, Matthew Chantry, Dwight Barkley

\section{To cite this version:}

Laurette S. Tuckerman, Matthew Chantry, Dwight Barkley. Patterns in Wall-Bounded Shear Flows. Annual Reviews of Fluid Mechanics, 2020. hal-02995006

\section{HAL Id: hal-02995006 https://hal.science/hal-02995006}

Submitted on 7 Jan 2021

HAL is a multi-disciplinary open access archive for the deposit and dissemination of scientific research documents, whether they are published or not. The documents may come from teaching and research institutions in France or abroad, or from public or private research centers.
L'archive ouverte pluridisciplinaire HAL, est destinée au dépôt et à la diffusion de documents scientifiques de niveau recherche, publiés ou non, émanant des établissements d'enseignement et de recherche français ou étrangers, des laboratoires publics ou privés. 


\section{Patterns in Wall-Bounded Shear Flows}

\section{Laurette S. Tuckerman ${ }^{1}$, Matthew Chantry ${ }^{2}$ and Dwight Barkley ${ }^{3}$}

${ }^{1}$ Laboratoire de Physique et Mécanique des Milieux Hétérogènes (PMMH), CNRS, ESPCI Paris, PSL Research University; Sorbonne Université, Univ. Paris Diderot,75005 France; email: laurette@pmmh.espci.fr

${ }^{2}$ Atmospheric, Oceanic and Planetary Physics, University of Oxford, Clarendon Laboratory, Parks Road, Oxford OX1 3PU, United Kingdom

3 Mathematics Institute, University of Warwick, Coventry CV4 7AL, United

Kingdom $\operatorname{xxxxxx} 0000.00: 1-26$

Copyright (c) 0000 by Annual Reviews. All rights reserved

\section{Keywords}

transition to turbulence, wall-bounded shear flows, turbulent-laminar pattern, directed percolation

\section{Abstract}

Experiments and numerical simulations have shown that turbulence in transitional wall-bounded shear flows frequently takes the form of long oblique bands, if the domains are sufficiently large to accommodate them. These turbulent bands have been observed in plane Couette, plane Poiseuille flow, counter-rotating Taylor-Couette flow, torsional Couette flow, and annular pipe flow. At their upper Reynolds-number threshold, laminar regions carve out gaps in otherwise uniform turbulence, ultimately forming regular turbulent-laminar patterns with a large spatial wavelength. At the lower threshold, isolated turbulent bands sparsely populate otherwise laminar domains and complete laminarization takes place via their disappearance. We review results for plane Couette flow, plane Poiseuille flow, and free-slip Waleffe flow focusing on thresholds, wavelengths and mean flows, with many of the results coming from numerical simulations in tilted rectangular domains that form the minimal flow unit for the turbulent-laminar bands. 


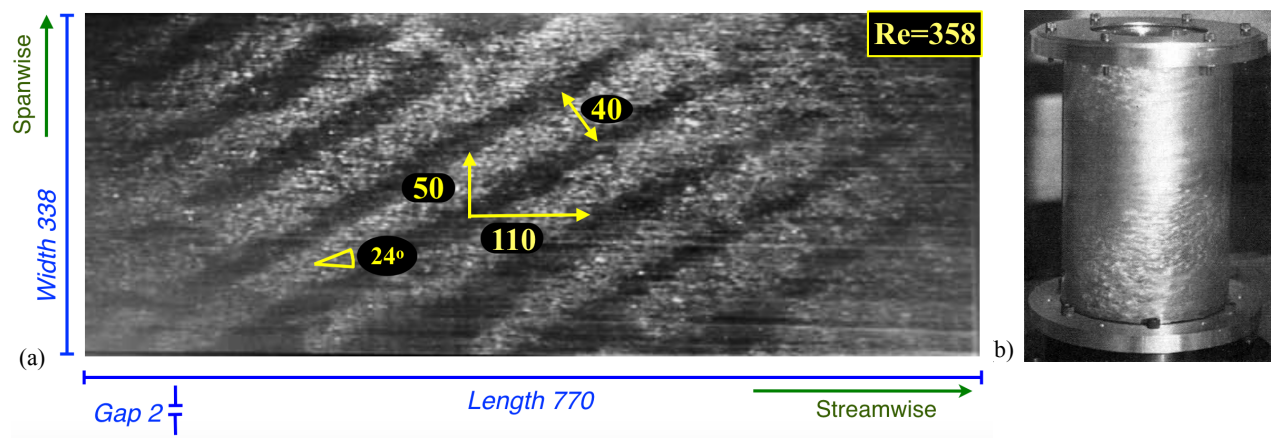

Figure 1

(a) Turbulent-laminar pattern in plane Couette flow experiment. Adapted from Prigent et al. (2003). (b) Turbulent spiral in Taylor-Couette flow experiment. From Coles \& van Atta (1966).

\section{Introduction}

The transition to turbulence in wall-bounded shear flows is often said to be subcritical, which calls to mind the famous quotation by Stanislaw Ulam "Using a term like nonlinear science is like referring to the bulk of zoology as the study of non-elephant animals" (Campbell et al. 1985). An essential feature of transitional turbulence is its spatial inhomogeneity, also called spatial intermittency. Classical transitions in configurations such as co-rotating TaylorCouette flow or Rayleigh-Bénard convection occur uniformly. There exists a threshold in Reynolds or Rayleigh number above which the laminar flow is linearly unstable and, after a relatively short time, the entire flow is occupied by a new state. For the wall-bounded shear flows which we will describe, this is not true. All of these flows are linearly stable in the Reynolds-number regime in which transition to turbulence occurs. For transition to take place, the flows must be subjected to a finite-amplitude perturbation. There exist various critical Reynolds numbers, one below which the asymptotic state is uniform laminar flow, and another above which it is uniform turbulence, separated by an intermediate range in which the asymptotic state is a mixture, more or less organized, of laminar and turbulent flow. The flows in this intermediate range are the subject of this article.

\section{Plane Couette flow: turbulent-laminar banded patterns}

Plane Couette flow is the flow between two parallel rigid plates that are separated by a fixed distance and that move at equal and opposite velocities. A particularly striking manifestation of spatial inhomogeneity in this system is a state consisting of persistent turbulent bands coexisting with nearly laminar regions, as shown in figure 1(a). Supplemental movie 1 shows the emergence of a turbulent-laminar banded pattern from uniform turbulence as $R e$ is lowered.

Such states occur in other similar shear flows. In particular they occur in counterrotating Taylor-Couette flow, the flow between concentric cylinders that rotate in opposite directions. Indeed, it was in counter-rotating Taylor-Couette flow that an oblique turbulent band - or in this geometry, a turbulent spiral - was first observed by Coles \& van Atta (1966), as seen in figure 1(b). This state was famously mentioned by Feynman (1964) and another depiction of it is included in the widely-cited encyclopedic survey of Taylor-Couette 
flow by Andereck, Liu \& Swinney (1986). (Because a translating frame is equivalent to a stationary one, only the relative velocity is significant in plane Couette flow. However, both relative and mean rotation are significant in Taylor-Couette flow. Consequently, flows with co-rotating and counter-rotating cylinders have quite different properties. In particular, a classic supercritical bifurcation occurs in parts of the co-rotating parameter space, while parts of the counter-rotating parameter space resemble plane Couette flow.)

Starting in the 1990s, it was shown that in a less confined geometry, the turbulent spiral is part of a regular alternating pattern which, moreover, also occurs in plane Couette flow. Tillmark \& Alfredsson (1992) used a looped belt to conduct pioneering experiments on transition in plane Couette flow. Using a similar device, Daviaud, Hegseth \& Bergé (1992) showed that turbulent spots grew into inclined patches, and suggested that these might be related to the turbulent Taylor-Couette spirals. Prigent, Grégoire, Chaté, Dauchot \& van Saarloos (2002) then carried out experiments with extremely large aspect ratios, i.e. spanwise and streamwise directions that were 338 and 770 times the half-gap for plane Couette flow and axial and azimuthal directions of 884 and 724 times the half-gap for counter-rotating Taylor-Couette flow. These large aspect ratios are necessary because the pattern wavelength is on the order of 40 times the half-distance between the plates. The bands are aligned obliquely (on the order of $24^{\circ}$ ) with respect to the streamwise direction. Both the wavelength and the angle depend, weakly but reproducibly, on the Reynolds numbers. This state can thus be called a turbulent-laminar banded pattern and it has been studied extensively since then.

Turbulent-laminar banded patterns were first simulated numerically by Barkley \& Tuckerman (2005a). A visualization of this pattern is shown in figure 2(a) via the kinetic energy at mid-gap. While the turbulent bands are oriented obliquely to the streamwise direction, within each turbulent region are structures primarily aligned with the streamwise direction. These structures correspond to the streamwise vortices and streaks known to be the essential building blocks of transitional shear turbulence (Hamilton, Kim \& Waleffe 1995, Waleffe 1997). The spanwise width of these structures is on the scale of the fluid gap. Streaks are clearly visible in the supplemental movie, which shows the streamwise velocity at the midplane. The red (positive) and blue (negative) undulating filamentary structures are the streaks.

A key feature of these simulations is the use of a periodic rectangular tilted domain, whose long direction is perpendicular to the bands, i.e. parallel to the wavevector of the pattern. Plates situated at $y= \pm h$ move at speed $U$ in equal and opposite directions at an angle $\theta$ from the $x$ axis. The laminar flow and Reynolds numbers are:

$$
\mathbf{u}_{\text {Cou }}^{\operatorname{lam}}=U \frac{y}{h} \mathbf{e}_{\text {strm }} \equiv U \frac{y}{h}\left(\cos \theta \mathbf{e}_{x}+\sin \theta \mathbf{e}_{z}\right) \quad R e=R e_{\text {Cou }} \equiv \frac{U h}{\nu}
$$

If $\theta=0$, there is no tilt and Eq. (1) reduces to the usual convention for plane Couette flow where $\mathbf{e}_{\text {strm }}=\mathbf{e}_{x}$. We call $y$ the vertical or wall-normal direction and $(x, z)$ the horizontal directions. Coordinates $x$ and $z$ will refer to the band-parallel and band-perpendicular directions; the streamwise and spanwise directions will be referred to as such. Velocity components $\mathbf{u} \equiv(u, v, w)$ will be specified where necessary. Where not otherwise specified, we will use non-dimensionalized variables such that $U=h=1$ and $R e$ will refer to $R e_{\mathrm{Cou}}$.

Figure 2(b,c,d) shows the construction principle for this domain. The idealized minimal flow unit, abbreviated MFU (Hamilton et al. 1995) of size $\left[L_{x}, L_{y}, L_{z}\right]=[4,2,6]$ is shown in figure 2(b). There is no tilt and its spanwise width $L_{z}=4$ is such as to contain just one pair of counter-rotating streamwise vortices, illustrated with red and blue colored bars.

www.annualreviews.org • 

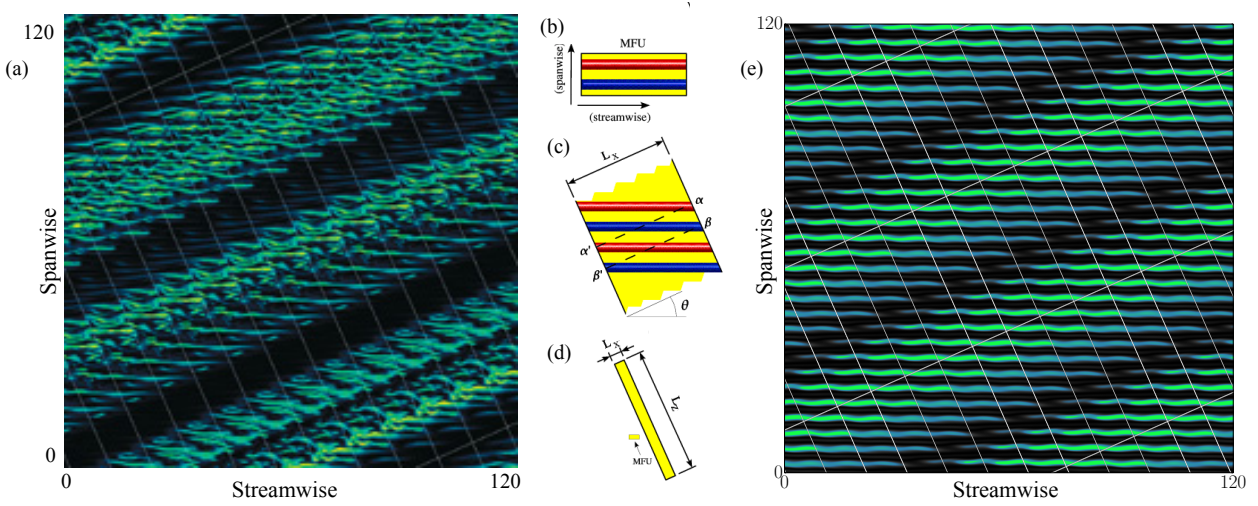

Figure 2

(a) Turbulent laminar-pattern for plane Couette flow at $R e=350$. calculated in a narrow rectangular tilted box. Shown is the kinetic energy at mid-gap of an instantaneous snapshot. The laminar regions are uniformly black, while the turbulent regions consists of sets of narrow horizontal streaks and vortices on the scale of the gap. The laminar regions are uniformly black, while the turbulent regions consist of narrow horizontal stripes on the scale of the gap. Adapted from Barkley \& Tuckerman (2005a). (b,c,d) Tilted box used for simulations. (e) Oblique unstable equilibrium state computed in tilted domain by Reetz, Kreilos \& Schneider (2019).

(The nominal spanwise width of a single vortex is the size of the fluid gap, which in nondimensional units is 2.) In order to sensibly impose periodicity in a tilted domain, streamwise vortices must meet adjacent ones in crossing a boundary, as illustrated in figure 2(c). To maintain a vortex-pair spacing of approximately 4 , the short direction of the domain $L_{x}$ must be related to the angle $\theta$ by $L_{x} \approx 4 / \sin \theta$. Shown here is a typical value of $24^{\circ}$ for which we take $L_{x}=10 \simeq 4 / \sin \theta=9.83$. Finally, the remaining horizontal dimension $L_{z}$ of the domain is taken to be long to contain one wavelength of the turbulent-laminar pattern, typically 40 half-gaps. This domain can be called the minimal band unit (MBU). The domain can also be prolonged, as in figure 2(d), to contain several repetitions of the pattern. The outlines of these domains, repeated periodically so as to tile figure 2(a), are seen as faint white lines.

Simulating turbulent-laminar patterns in tilted computational domains with a single long direction has two purposes. The first is the obvious reduction in the computational resources required in comparison to those required for domains with two large directions. The second is that such simulations capture the minimal, or near-minimal conditions necessary for turbulent-laminar patterns to form and this allows more direct access to the mechanisms underlying these patterns.

Figure 2(e) shows an unstable equilibrium solution computed by Reetz, Kreilos \& Schneider (2019) which greatly resembles the instantaneous pattern on the left. Following the first calculation of a non-trivial equilibrium by Nagata (1990), a large number of unstable equilibria (called exact coherent structures in the context of wall-bounded shear flows) have been computed (e.g., Gibson, Halcrow \& Cvitanović 2009). The scenario deduced by Reetz et al. (2019) shows that the equilibrium in figure 2(e) is connected to the Nagata solution via two successive bifurcations. Oblique equilibria have also been calculated by high-Re asymptotic methods by Deguchi \& Hall (2015). Unstable equilibria are thought 
(a) $\mathrm{Re}=330$

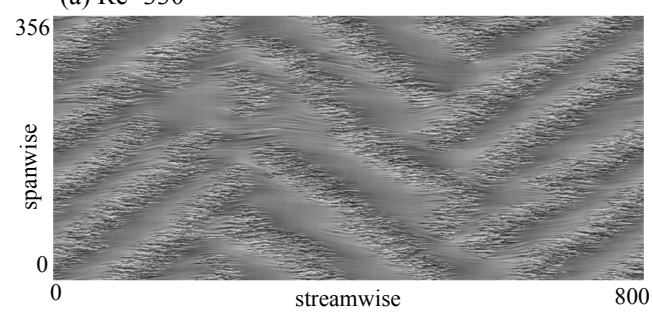

(b) $\mathrm{Re}=393$

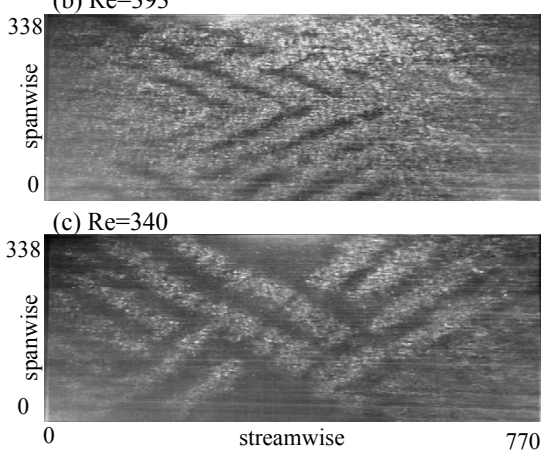

Figure 3

Competition in plane Couette flow between turbulent-laminar bands with opposite angles. (a) Streamwise velocity in midplane computed by Duguet et al. (2010) in a domain oriented along the streamwise and spanwise directions. (b,c) Plane Couette experiment of Prigent et al. (2003). The wavelength increases as $R e$ decreases.

to play an underlying role in organizing turbulence (Cvitanović \& Eckhardt 1989, Kawahara \& Kida 2001). Schneider, Marinc \& Eckhardt (2010) have extended these ideas to compute edge states which are spatially localized within large domains and mildly chaotic. We shall not focus further on equilibria; see Kawahara, Uhlmann \& Van Veen (2012).

Domains large in both the streamwise and spanwise directions, while less economical, can capture further features of the systems such as competition between different angles and wavelengths. Figure 3 shows turbulent-laminar banded patterns with competing angles in plane Couette flow from simulations by Duguet, Schlatter \& Henningson (2010) and from experiments by Prigent, Grégoire, Chaté \& Dauchot (2003). The competition between bands at opposite angles was studied by these authors and by Hegseth, Andereck, Hayot \& Pomeau (1989) and Goharzadeh \& Mutabazi (2001, 2008) in terms of diffusive phase dynamics and Ginzburg-Landau equations.

Most of the simulations presented in this section were obtained using the spectralelement/Fourier code Prism (Henderson \& Karniadakis 1995) to integrate the Navier-Stokes equations on grids containing 6-12 points per unit length in the horizontal directions and $15-20$ in the wall-normal direction in a domain tilted at $24^{\circ}$. We will also present some simulations in large domains whose sides are aligned with the streamwise and spanwise directions.

Turbulent-laminar patterns are associated with large-scale and reproducible mean flows, as can be expected since they break the spatial homogeneity in the streamwise and spanwise directions. Entitling their article "Progress report on a digital experiment in spiral turbulence" to highlight their then-groundbreaking experimental techniques, Coles \& van Atta (1966) sketched the form of the mean flow at midgap between the two cylinders in their Taylor-Couette apparatus; see figure 4(a). Results in figure 4(b) from our full numerical computations in plane Couette flow show the mean flow to be somewhat different, but also emphasize the importance of strong flow parallel to the band boundaries. Duguet \& Schlatter (2013) show that this along-band flow is a simple consequence of the incompressibility of the large-scale flow. 

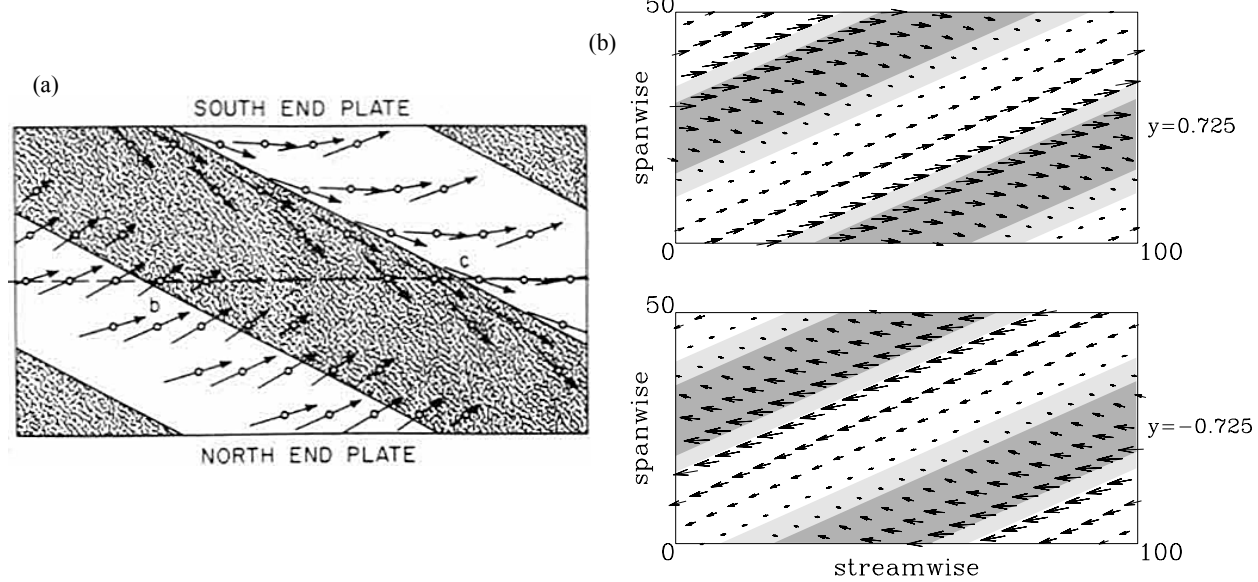

Figure 4

Horizontal views of flow accompanying turbulent-laminar banded pattern. (a) Sketch from experimental data at mid-plane of Taylor-Couette flow in Coles \& van Atta (1966). (b)

Computations of plane Couette flow, above and below mid-plane, adapted from Barkley \&

Tuckerman (2007). Turbulent regions are shown in grey and laminar regions are shown in white.

Both show prominent band-parallel flow at the turbulent-laminar boundaries.

A more complete view of the mean structure of a turbulent-laminar pattern is presented in figure 5. We use \langle\rangle to denote averaging in time and along the band direction $x$, yielding functions which depend on the wall-normal direction $y$ and the band-perpendicular direction $z$. The deviation from laminar Couette flow is plotted in a periodically prolonged domain. The centers of two adjacent turbulent bands are located at $z=-20$ and $z=20$, as seen in the turbulent kinetic energy $E_{\text {turb }}$. The intervening laminar region is centered on $z=0$. The top plot shows the along-band flow $\langle u\rangle$ (into and out of the plane shown), which is strong at the interfaces. The flow along the bands is accompanied by a large scale circulation around the turbulent bands, although we emphasize that this streamfunction $\langle\psi\rangle$ corresponds to the deviation from laminar Couette flow. For this periodic pattern, the mean flow can be approximated to high accuracy as the sum of only three trigonometric functions in the band-perpendicular direction $z$ :

$$
u(y, z) \approx u_{0}(y)+u_{c}(y) \cos (2 \pi z / \lambda)+u_{s}(y) \sin (2 \pi z / \lambda)
$$

where $\lambda$ is the wavelength, here 40 half-gaps, and $u$ represents $\langle u\rangle$ or $\langle\psi\rangle$.

Figure 6 illustrates various regimes of turbulent-laminar patterns as a function of Reynolds number. Spatiotemporal diagrams have been produced by sampling simulations in tilted domains along a line in the (long) band-perpendicular direction $z$. The Reynolds number or the domain size is also evolved over the course of the simulation. Figure 6(a) shows the evolution to the patterned state as the Reynolds number is decreased. Time evolves upward and as it does $R e$ is decreased in steps as shown at the right of the figure. At $R e=500$ the flow is in a state of uniform, or featureless turbulence. Following a decrease to $R e=350$, a distinct pattern forms with three turbulent bands alternating with regions of quasi-laminar flow. As $R e$ is further decreased, one turbulent band is lost at $R e=310$ 


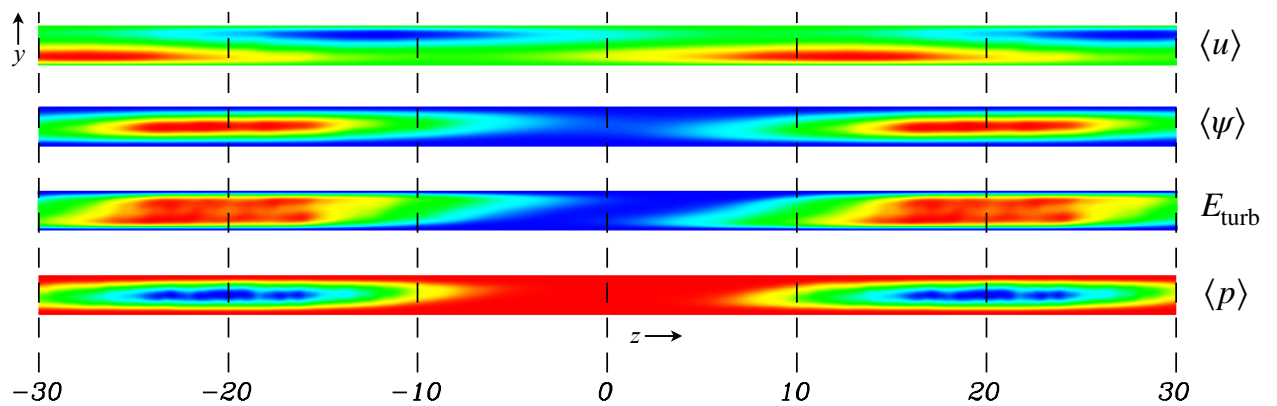

Figure 5

Mean flow of a turbulent-laminar pattern at $R e=350$, with the average taken over time and the band-parallel direction, and laminar plane Couette flow subtracted. The centers of the turbulent bands are at $z= \pm 20$, the center of the laminar region at $z=0$ and the boundaries at $z \approx \pm 10$. The band-parallel flow $\langle u\rangle$ is strongest at the boundaries. The flow in the $(y, z)$ plane is represented by a streamfunction $\langle\psi\rangle$, which shows circulation around the turbulent band, characterized by high turbulent kinetic energy $E_{\text {turb }}$ and low pressure $\langle p\rangle$. Color ranges for each field from blue to red: $\langle u\rangle[-0.4,0.4],\langle\psi\rangle[0,0.09], E_{\text {turb }}[0,0.4],\langle p\rangle[0,0.007]$. From Barkley \& Tuckerman (2007).

and a second is lost at $R e=300$, leaving a single band that subsequently fully relaminarizes at $R e=290$. The general trend shown in Figure 6(a) is robustly observed with decreasing Reynolds number, but the details will vary with realization. This Reynolds number scan is coarse; more precise thresholds and means of determining them are described later. In particular, patterned states that appear to be stable may ultimately revert to laminar flow on very long time scales. Figure 6(b) shows the reverse transition from a turbulent-laminar banded pattern to uniform turbulence as the Reynolds number is increased from 350 to 420. Figure 6(c) shows that if the Reynolds number is fixed at 350 and the domain size is increased, a turbulent band repeatedly splits in order to retain a wavelength of about 40 . The same procedure for $R e=300$ in (d) shows a single isolated band, independent of the domain size. This localized band is expected to revert to laminar flow on some very long time scale. In (e), $R e=410$ and the domain size is fixed at 40 . Laminar and turbulent patches repeatedly appear and disappear. In all of the cases shown in figure 6 , when laminar patches appear, the intensity of the turbulence within the bands increases, keeping the total turbulent intensity approximately constant.

A quantitative characterization of the pattern is provided by the line graphs to the left of figures 6(a), (b), and (e). These show the time-dependent moduli $\left|w_{\lambda}\right|$ of the coefficients of the one-dimensional spatial Fourier transform (in the band-perpendicular direction) of the spanwise velocity at mid-gap averaged over intervals of $\Delta T=500$. These provide a good characterization of the patterns, with significant $\lambda=40$ and $\lambda=60$ components at appropriate times and it is this information that has been used to define a threshold. Figure 7 (a) presents the Reynolds-number dependence of the spectral coefficient $\left|\hat{w}_{\lambda=40}\right|$, where averages have been taken over long time series such as the one in figure 6(e). A clear transition is seen near $R e=430$, which we take to be the threshold between uniform turbulence and turbulent-laminar bands in plane Couette flow in our narrow tilted rectangular domain. Much of section 5 will be devoted to characterizing the lower threshold that separates laminar flow from the presence of turbulence. Figure 7 (b) constructs probability distribution 

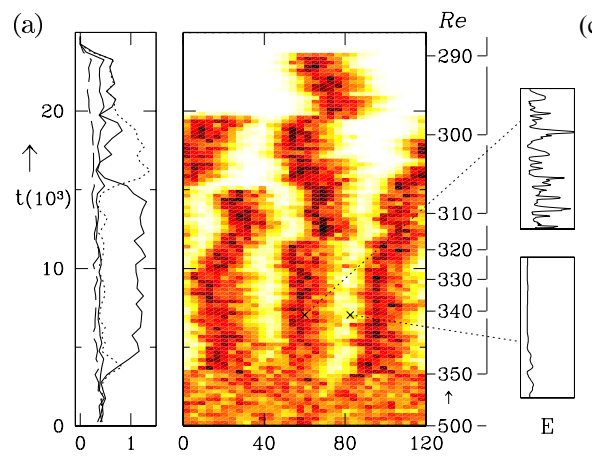

(c)

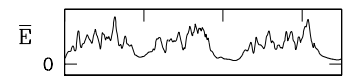

(e)
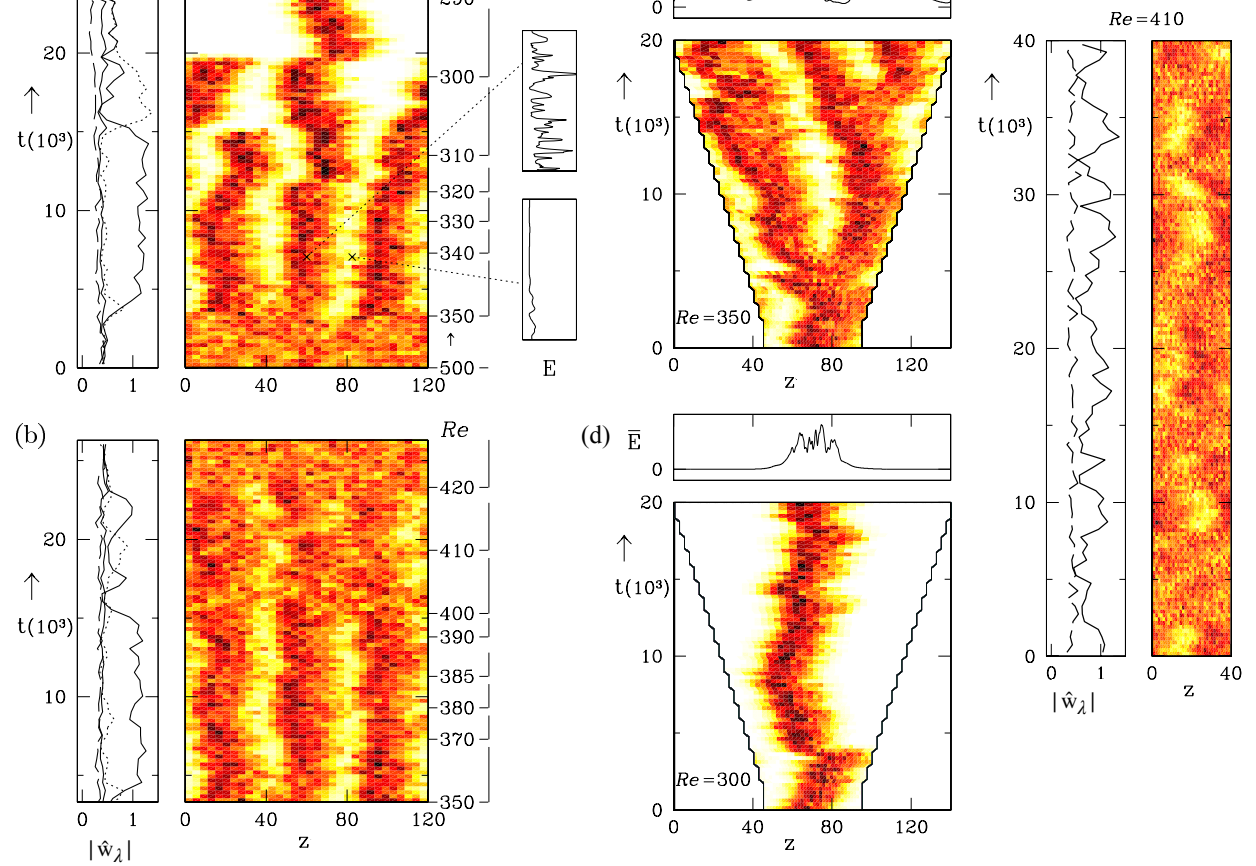

Figure 6

Different regimes of turbulent-laminar banded patterns in plane Couette flow. Spatiotemporal diagrams are constructed by sampling the kinetic energy at 32 points along a line at the mid-gap $y=0$ in the long direction $z$, averaging over temporal windows of length $T=500$ and using colors varying from white (zero; laminar flow) to black (turbulent). For (a,b), the domain length is $L_{z}=120$. In (a), we start with uniform turbulence and lower the Reynolds number from $R e=500$. Three lighter laminar patches appear when $R e=350$, leading to a wavelength of 40 . Near 310 , one of the patches disappears, leading to a wavelength of 60 , and near 300 , another disappears, leading to a single patch, which disappears at 290. Plots to the right show detailed evolution of the kinetic energy within the temporal window in turbulent and laminar regions. In (b), the initial condition is the turbulent-laminar banded pattern at $R e=350$, which evolves to uniform turbulence when the Reynolds number is increased. In (c) and (d), the domain length is increased in small discrete steps from $L_{z}=50$ to $L_{z}=140$. For $R e=350$ (c), the turbulent region splits to retain an approximate wavelength of 40 , while for $R e=300$ (d), its width remains constant, corresponding to a single localized band. Plots above (c) and (d) of the final instantaneous turbulent kinetic energy show that for $R e=350$, the turbulent kinetic energy remains above zero throughout the domain, whereas for $R e=300$ it is zero in the laminar region. In (e), with $R e$ fixed at the intermediate value of 410 and $L_{z}$ fixed at 40 , the state remains poorly defined, with turbulent patches appearing and disappearing. Plots to the left of (a), (b), and (e) show the variation in time of the one-dimensional spatial Fourier components of the spanwise velocity, with the solid, dotted, short-dashed, and long-dashed curves corresponding to the $\lambda=40$, $\lambda=60, \lambda=120$, and uniform components.

functions from these long timeseries by averaging over intervals of $\Delta T=500$. At $R e=500$, when the turbulence is uniform, the most probable value of $\left|\hat{w}_{\lambda=40}\right|$ is 0 . At $R e=410$, the distribution undergoes an inflection and by $R e=350$, when a turbulent-laminar pattern 

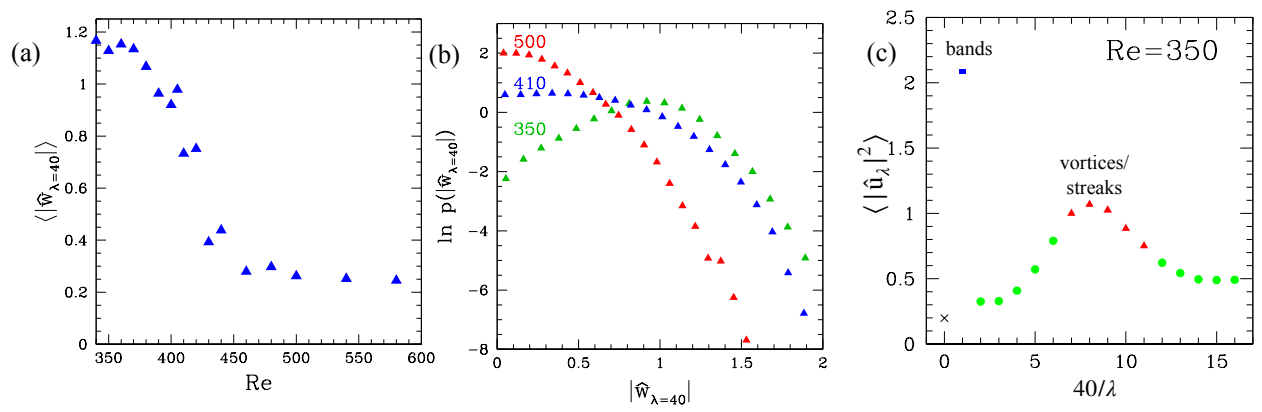

Figure 7

Modulus $\left|\hat{w}_{\lambda=40}\right|$ of the $\lambda=40$ Fourier component in the band-perpendicular direction of the spanwise velocity at mid-gap, such as shown in figure 6(e). (a) Average over timeseries. A transition between uniform turbulence and a pattern is seen near $R e=430$. (b) Logarithm of probability distribution function for a uniformly turbulent flow at $R e=500$, an intermittent flow at $R e=410$, and a turbulent-laminar patterned flow at $R e=350$. The most probable value is zero for uniform turbulence but has a finite value for a patterned flow. (c) Time-averaged band-perpendicular square Fourier component of the streamwise velocity $\left|\hat{u}_{\lambda}\right|^{2}$ for $R e=350$. Dominant coefficient corresponding to bands has wavelength $\lambda=40$, while coefficients corresponding to streamwise vortices and streaks have $3.6 \leq \lambda \leq 5.7$. Adapted from Tuckerman \& Barkley (2011).

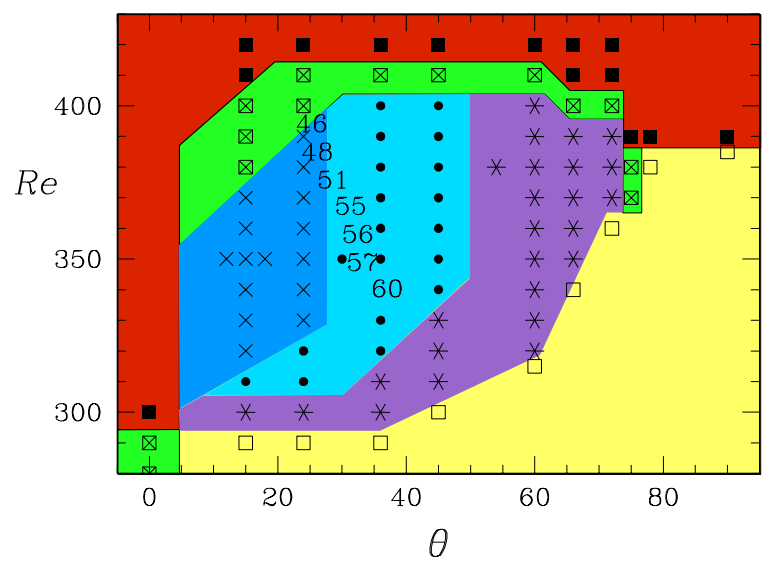

Figure 8

Survey of turbulent-laminar patterned regimes in our computations of plane Couette flow in a narrow tilted rectangular domain as a function of imposed angle $\theta$ and Reynolds number $R e$.

Uniform turbulence (solid squares, red), intermittent (green, crosses in squares), turbulent-laminar patterns with wavelength 40 (blue, crosses) or 60 (light blue, dots), localized states (stars, purple), laminar Couette flow (hollow squares, yellow). Numbers show wavelengths found in experiments of Prigent et al. (2003) at indicated values of $\theta$ and Re. From Tuckerman \& Barkley (2011).

with $\lambda=40$ is robust, the most probable value is non-zero. Finally, figure 7 (c) presents the entire Fourier spectrum at $R e=350$, contrasting the coefficients corresponding to the bands, with $\lambda=40$, with those of the much smaller structures with $\lambda \approx 4$, twice the gap of size 2. These smaller structures, as seen in figure $2(\mathrm{a})$, consist of streamwise vortices 
(wall-normal and spanwise flow) and streaks (spanwise dependence of the streamwise velocity). At mid-gap their mean spanwise velocity is zero and hence we show the streamwise components $\left|\hat{u}_{\lambda}\right|$.

Finally, figure 8 presents a survey of the regimes that are seen in minimal band units as a function of imposed angle $\theta$ and Reynolds number. The experiments of Prigent et al. (2003) show wavelengths (in the band-perpendicular direction) that increase from 46 to 60 half-gaps and angles that increase from $25^{\circ}$ to $37^{\circ}$ as the Reynolds number is decreased, as can also be seen in figure $3(\mathrm{~b}, \mathrm{c})$. Because the angle is imposed in tilted MBU domains, simulations can compute patterns with a wide range of both angles and wavelengths. Most of these are presumably unstable when placed in a larger, less constrained domain, since they have not been observed. For $15^{\circ} \leq \theta \leq 72^{\circ}$, we use a tilted domain whose dimensions are $[4 / \sin \theta, 2,120]$ and find periodic or localized bands. Simulations in a non-tilted domain with a long streamwise length of 220 and a short spanwise direction of length 4 (corresponding to $90^{\circ}$ ) show direct laminarization at $R e \approx 400$ after a very short-lived transient pattern. In contrast, simulations with a long spanwise length of 120 and a short streamwise length of 10 (corresponding to $0^{\circ}$ ) show repeated nucleation of turbulent regions which persists to Reynolds numbers as low as $R e \approx 200$; see Barkley \& Tuckerman (2005b).

\section{Poiseuille flow: two superposed shear layers}

Turbulent-laminar banded patterns also exist in plane Poiseuille flow, sometimes called channel flow - the flow between two parallel rigid plates maintained at a constant distance and driven by an imposed streamwise pressure gradient or flux; see figure 9. Banded patterns were observed first numerically in plane Poiseuille flow by Tsukahara and coworkers $(2005,2006)$ and experimentally by Hashimoto, Hasobe, Tsukahara, Kawaguchi \& Kawamura (2009), with later simulations by Brethouwer, Duguet \& Schlatter (2012), Fukudome \& Iida (2012), Tuckerman, Kreilos, Schrobsdorff, Schneider \& Gibson (2014), Xiong, Tao, Chen \& Brandt (2015), Kushwaha, Park \& Graham (2017) and Tao, Eckhardt \& Xiong (2018).

The properties of transitional plane Poiseuille flow resemble those of plane Couette flow. However, the similarities are somewhat masked by the trivial fact that the two flows effectively use different non-dimensionalization. Plane Poiseuille flow can be viewed as the superposition of two shear layers of opposite sign. Thus the characteristic length scale in Poiseuille flow - the half-channel height - is the full height of one of these shear layers. In contrast, the half-channel height in the Couette flow convention is half its shear-layer height. In addition, several different conventions are used for the velocity scale in plane Poiseuille flow. Here we consider the system driven by an imposed flux, rather than imposed pressure gradient. Hence the mean streamwise velocity $U_{\text {bulk }}$ is known. We use as a velocity scale $U \equiv 3 U_{\text {bulk }} / 2$, which is the centerline velocity of laminar flow with mean velocity $U_{\text {bulk }}$. The velocity scale, $U$, is then the difference between the maximum and minimum velocity of laminar Poiseuille flow. However, in Couette flow the velocity scale used is half the difference between the velocities of the two walls. As a result, in terms of the shear, both the length scale and the velocity scales used for nondimensionalization in plane Poiseuille flow are twice those used in plane Couette flow. Thus, roughly speaking, one should expect values of $R e_{\text {Poi }}$, as conventionally defined, to differ from values of $R e_{\text {Cou }}$ by a factor of four:

$$
\mathbf{u}_{\mathrm{Poi}}^{\operatorname{lam}}=U\left(1-\frac{y^{2}}{h^{2}}\right) \mathbf{e}_{\mathrm{strm}} \quad R e_{\mathrm{Poi}} \equiv \frac{U h}{\nu}=\frac{4\left(\frac{U}{2}\right)\left(\frac{h}{2}\right)}{\nu} \approx 4 R e_{\mathrm{Cou}}
$$



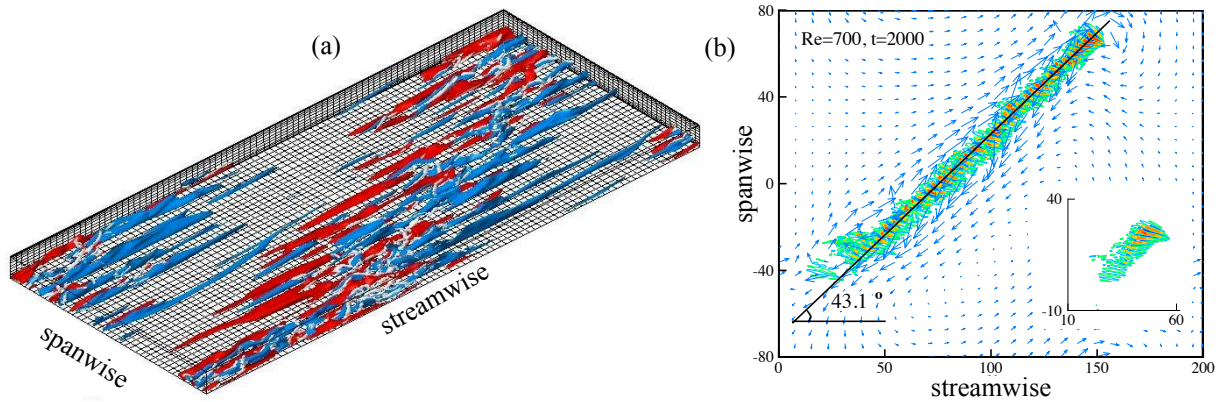

Figure 9

Turbulent bands in plane Poiseuille flow. (a) Three-dimensional view of deviation from laminar flow of streamwise velocity at $R e_{\mathrm{Poi}}=1140$ in lower half of $[51.2,2,22.5]$ domain from Tsukahara et al. (2006). (b) wall-normal velocity (colors) and horizontal mean flow (arrows) at $R e_{\text {Poi }}=700$ in a $[200,2,160]$ domain from Xiong et al. (2015). The inset is the initial condition. All lengths are in units of the half-gap, $h$; time, $t$, is in advective units, $h / U$, where $U$ is $3 / 2$ of the bulk velocity. Panels adapted with permission from

where $R e_{\text {Cou }}$ uses plane Couette conventions based on each single-sign shear layer.

Plane Poiseuille flow also differs from plane Couette flow in its symmetries. Plane Couette flow possesses centro-symmetry, i.e. simultaneous reflection in the streamwise and wall-normal directions. In contrast, although plane Poiseuille flow has reflection symmetry in the wall-normal direction, it is not symmetric between the upstream and downstream directions. Therefore turbulent-laminar bands in plane Couette flow are stationary, at least on short time scales, while in plane Poiseuille flow they generically travel in the streamwise direction since there is no symmetry to keep them stationary.

Figure 10 shows results from simulations for plane Poiseuille flow in a tilted MBU domain with dimensions $[10,2,40]$. The simulations use the pseudospectral Chebyshev/Fourier Channelflow 2.0 code (Gibson, Reetz, Azimi, Ferraro, Kreilos et al. 2019) with 12 points per unit horizontal length and 32 per unit wall-normal length. The protocol is similar to that used in the previous plane Couette simulations - the Reynolds number is decreased in discrete steps during the simulation. Results are shown in a frame of reference moving at the mean velocity $U$. Figure 10(a) shows that, starting from uniform turbulence and decreasing the Reynolds number, the approximate threshold for appearance of the bands is $R e_{\text {Poi }} \approx 1900=475 \times 4$; that for localized bands is $R e_{\text {Poi }} \approx 1300=325 \times 4$; and that for relaminarization is $R e_{\text {Poi }} \approx 800=4 \times 200$ in the MBU. Rather than a typical wavelength of 40 half-gaps, the wavelength at onset is 20 half-gaps, i.e. 40 quarter-gaps, the natural comparison to use with plane Couette flow. The travelling character of the turbulent-laminar patterns is evident. Patterns travel more quickly than the mean flow for $R e_{\text {Poi }} \lesssim 1100$ and more slowly for $R e_{\text {Poi }} \gtrsim 1100$; the velocities range from approximately $0.03 U$ to $-0.03 U$. Figure 10(b,c,d) also shows cross-sections of time and band-direction averaged fields; see also Tsukahara et al. (2005). The streamwise velocity, alternates between a parabolic and flattened profile, the streamfunction shows two superposed elongated recirculation cells, and the turbulent kinetic energy is concentrated near the two bounding walls.

We now consider the various forces which must be in equilibrium to maintain a statistically permanent turbulent-laminar banded pattern, and which dominate in the turbulent 

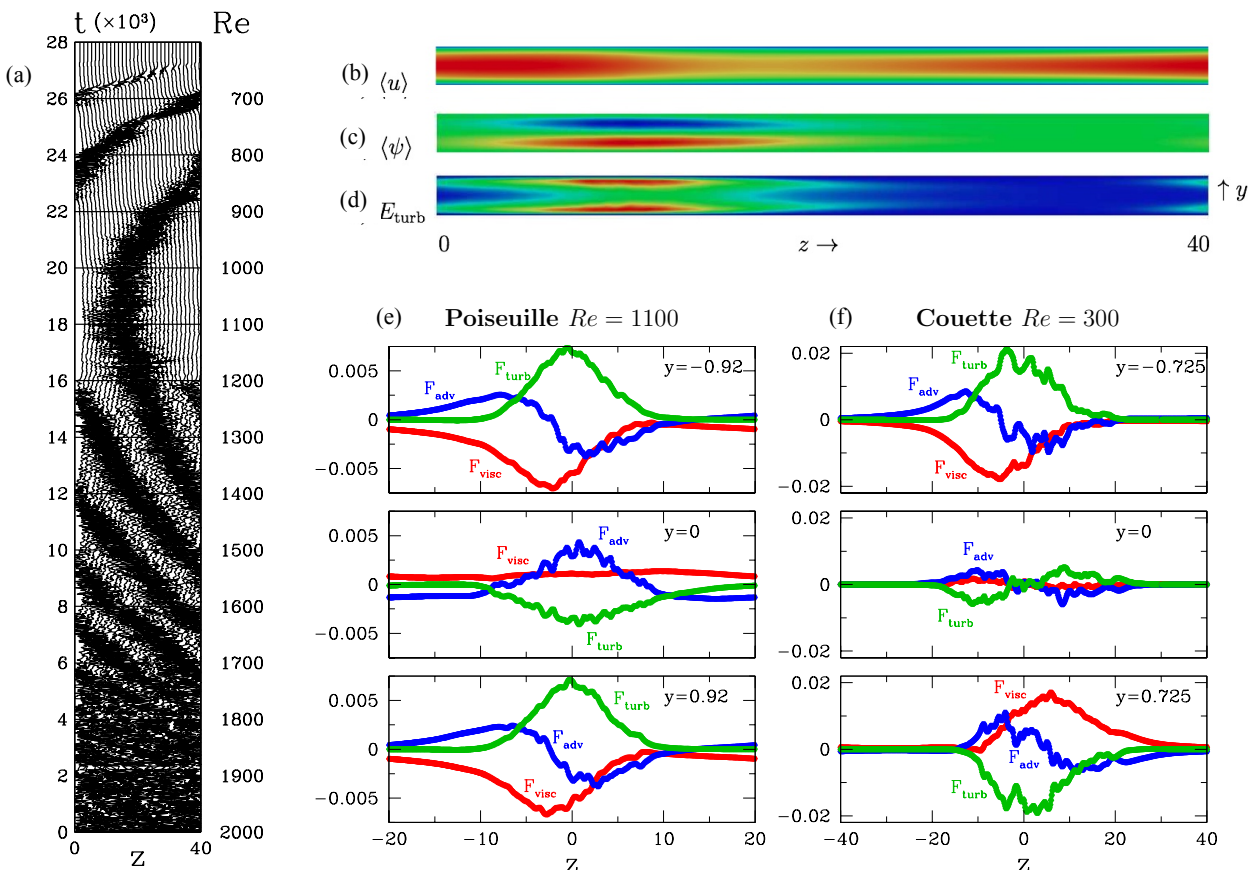

(e) Poiseuille $R e=1100$

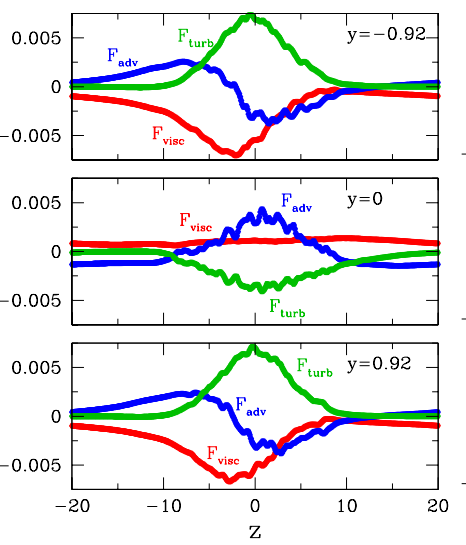

(f) Couette $R e=300$

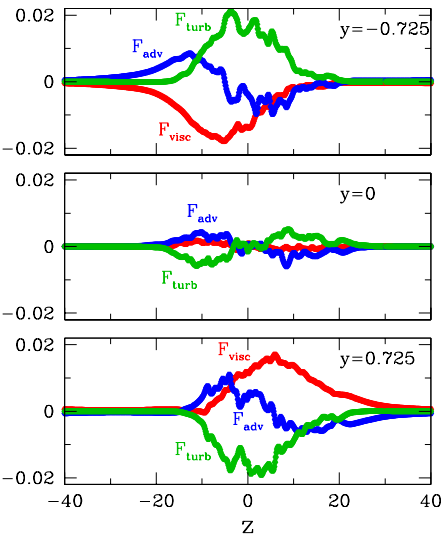

Figure 10

(a) Evolution of simulation of plane Poiseuille flow in long narrow tilted rectangular channel in the frame of the streamwise bulk velocity. The simulation is initialized with uniform turbulence at $R e_{\text {Poi }}=2000$ and the Reynolds number is decreased in steps of 100. Spanwise velocity timeseries at 32 points along a line at $y=0.8$ are plotted. Laminar bands with wavelength 20 emerge at around $R e_{\text {Poi }} \approx 1900$, with wavelength increasing to 40 at $R e_{\text {Poi }} \approx 1200$ and turbulence disappearing at $R e_{\mathrm{Poi}} \approx 800$. The pattern moves more slowly than the bulk velocity for $R e_{\text {Poi }} \gtrsim 1100$ and more quickly for $R e_{\text {Poi }} \lesssim 1100$. (b,c,d) Temporally and band-averaged fields for $R e=1100$. (b) Streamwise velocity $\langle u\rangle$ (including laminar profile) alternates between a parabolic profile in $y$, in which the maximum value of $\langle u\rangle$ is high, and a flattened profile, in which it is low. (c) Streamfunction $\langle\psi\rangle$ in the $(y, z)$ plane shows two superposed elongated recirculating cells. (d) Turbulent kinetic energy $E_{\text {turb }}$ shows that the turbulence is localized near the walls, at which the shear is highest. (e,f) Streamwise Reynolds stress (green), advective (blue), and viscous (red) forces as a function of $z$ at three $y$ locations for the mean flow associated with turbulent-laminar band in Poiseuille flow (e) at $R e_{\mathrm{Poi}}=1100$ and in Couette flow (f) at $R e_{\mathrm{Cou}}=300$. For both flows, $F_{\text {turb }}$ acts in the same direction as the laminar profile, while $F_{\text {visc }}$ is in the opposite direction. The balance of forces is very similar for the $y>0$ and $y<0$ sections of Poiseuille flow and the $y<0$ section of Couette flow. The force balance for the $y>0$ and $y<0$ sections of Couette flow are related by centro-symmetry. All lengths are in units of the half-gap, $h$; time, $t$, is in advective units, $h / U$, where $U$ is $3 / 2$ of the bulk velocity. From Tuckerman et al. (2014).

or laminar regions. We write the averaged Navier-Stokes equation

$$
0 \approx \underbrace{-\langle(\tilde{\mathbf{u}} \cdot \nabla) \tilde{\mathbf{u}}\rangle}_{F_{\text {turb }}}+\underbrace{-\left\langle\mathbf{u}^{\text {lam }} \cdot \nabla\left(\mathbf{u}-\mathbf{u}^{\text {lam }}\right)\right\rangle}_{F_{\text {adv }}}+\underbrace{\frac{1}{R e} \nabla^{2}\left\langle\mathbf{u}-\mathbf{u}^{\text {lam }}\right\rangle}_{F_{\text {visc }}}
$$

Equation (4) omits the largest forces, which balance to maintain the laminar flow, as well as some of the negligibly smallest forces. We have projected onto the streamwise direction 
to define $F_{\text {turb }}$, the turbulent or Reynolds-stress force; $F_{\text {adv }}$, expressing the dominant advection by the laminar flow; and $F_{\text {visc }}$, the viscous force countering curvature. Figure 10 shows these forces at three wall-normal locations as a function of $z$ for a turbulent band for both Poiseuille and Couette flow. The relation between the forces above and below mid-gap respect the symmetries of the Poiseuille and Couette configurations, while the relation between the forces in the Poiseuille and Couette flows confirms the interpretation of Poiseuille flow as two superposed Couette flows. Recalling that $z$ has a component in the streamwise direction and given the signs of Poiseuille and Couette flow in the upper and lower halves of the channel, $F_{\text {turb }}$ mostly acts to accelerate the fluid in the streamwise direction and $F_{\text {visc }}$ to oppose it. $F_{\text {adv }}$ changes sign as the band is traversed. The Couette turbulent band is localized: in the laminar region where $F_{\text {turb }}=0$, we also have $F_{\text {adv }}=F_{\text {visc }}=0$. The Poiseuille turbulent band, though, is bordered by regions in which $F_{\text {turb }}=0$ but $F_{\text {adv }}$ and $F_{\text {visc }}$ are equal and opposite, though small.

An interesting feature of plane Poiseuille flow is that a localized perturbation may evolve into an isolated oblique turbulent bands by extending from only one of its endpoints, as in figure 9(b) from Xiong et al. (2015). This is another manifestation of the asymmetry between the upstream and downstream streamwise directions of plane Poiseuille flow: unlike in plane Couette flow, here the two ends of a single band experience a different relationship to the streamwise flow. Spreading is observed to start at $R e_{\mathrm{Poi}}=660$. Above this Reynolds number localized turbulent bands increase steadily in length in sufficiently large domains, or sustain themselves in a cycle of band extension and breakup in periodic domains (Xiong et al. 2015, Tao et al. 2018, Kanazawa 2018).

\section{Waleffe flow: role of the walls}

An important question is the role of walls in wall-bounded shear flows, assumed in their very name. The necessity for rigid walls was questioned by Waleffe (1997), who derived his classic Self-Sustaining Process not from wall-bounded plane Couette flow, but from a simplified version in which the no-slip boundary conditions $u=v=w=0$ at $y= \pm 1$ are replaced by free-slip conditions $\partial_{y} u=v=\partial_{y} w=0$. The flow is still confined between boundaries, but is not driven by wall motion or by a pressure gradient, but instead by an imposed bulk force varying sinusoidally in the wall-normal direction $y$.

Figure 11(a) shows the correspondence between plane Couette flow and Waleffe flow. In plane Couette flow, the laminar profile is straight, while the mean turbulent flow takes a sigmoidal form with sharp changes near the boundaries and is nearly linear in the interior, corresponding to constant mean shear. In contrast, in Waleffe flow the laminar profile is sinusoidal while the mean turbulent profile is roughly linear corresponding to a constant shear. If the domain of Waleffe flow is taken to be the interior of plane Couette flow, as in figure 11(a), then Waleffe flow can be viewed as modeling only this region, without the near-wall regions (Chantry, Tuckerman \& Barkley 2016). Figure 11(a) indicates a specific scaling based on this correspondence: the height $H$ of the Waleffe domain is 0.625 times the height $h$ of the plane Couette domain and the maximum speed $V$ of the Waleffe profile is correspondingly 0.625 times the speed $U$ of the plane Couette profile. This height, that of the inner near-linear portion of the mean turbulent plane Couette flow profile, is not universal but varies slowly with $R e$ in the transition region. We define a Reynolds number 

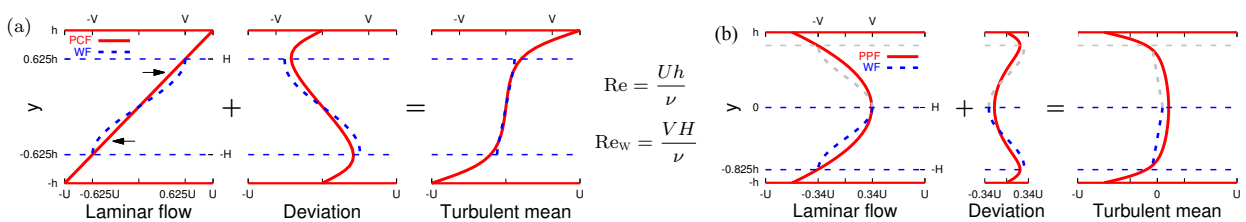

Figure 11

Correspondence between flows with rigid and with free-slip walls. (a) Plane Couette flow and Waleffe flow. The wall-normal bounds $\pm H$ of the Waleffe flow domain and its velocity extrema $\pm V$ are matched to those of the interior portion of the turbulent mean profile of plane Couette flow with wall-normal bounds $\pm h$ and velocity extrema $\pm U$. (b) Plane Poiseuille flow and its corresponding free-slip version as two superposed Waleffe flows. From Chantry et al. (2016).

Re for Waleffe flow, based on the correspondence with plane Couette flow, i.e.

$$
\mathbf{u}_{\mathrm{Wal}}^{\operatorname{lam}}=V \sin \left(\frac{\pi}{2} \frac{y}{H}\right) \mathbf{e}_{\mathrm{strm}} \quad R e \equiv \frac{U h}{\nu}=\frac{V / 0.625 \times H / 0.625}{\nu}
$$

where the notation is defined in figure 11. Simulations are carried out by adapting Channelflow 2.0 (Gibson et al. 2019) for the free-slip conditions, on a grid with 12 points per horizontal unit length and 15 per wall-normal unit length.

Waleffe flow undergoes the same sequence of transitions as plane Couette flow as the Reynolds number is decreased, from uniform turbulence through regular turbulent-laminar bands, then isolated and fragmented bands, and finally to laminar flow. Turbulent-laminar patterns exist in Waleffe flow approximately for $R e \in[250,640]$, a wide range which encompasses the corresponding range for plane Couette flow. Figure 12 compares the patterns for both flows. Horizontal views of the instantaneous flows and as well as vertical views of the averaged flows show the marked resemblance between the patterns. (Model Waleffe flow (MWF), also shown on this figure, will be discussed later.)

The free-slip version of plane Poiseuille flow is constructed explicitly as a superposition of two free-slip Couette flows, or equivalently as a free-slip channel driven by a body force with $y$ dependence $\cos (\pi y / H)$, as shown in figure 11(b). There are two provisos. First, rigidwall plane Poiseuille flow has two boundary layers that are to be "clipped", rather than the four that would exist in two superposed rigid-wall plane Couette flows, leading to slightly different scalings. Second, a Tollmien-Schlichting-like eigenmode which would otherwise be unstable at transitional Reynolds numbers must be suppressed. With the appropriate scaling and Reynolds-number definition, turbulent-laminar bands are then present in freeslip plane Poiseuille flow over the range [700,1800], close to the range found for the rigid case; see figure 10(a). The temporally and spatially band-parallel averaged flows in figure 12 highlight the resemblance between these flows in the rigid-wall and free-slip versions, as well as the interpretation of plane Poiseuille flow as two superposed plane Couette flows; see also figures 5 and 10, in which the wall-normal direction is not stretched.

Other ideas for simulating fully turbulent channel flow at higher Reynolds numbers in the interior region only have been proposed by Podvin \& Fraigneau (2011) and Mizuno \& Jiménez (2013), who substitute for the no-slip boundary condition at the wall a synthetic velocity field imposed at an interior, off-wall boundary. 

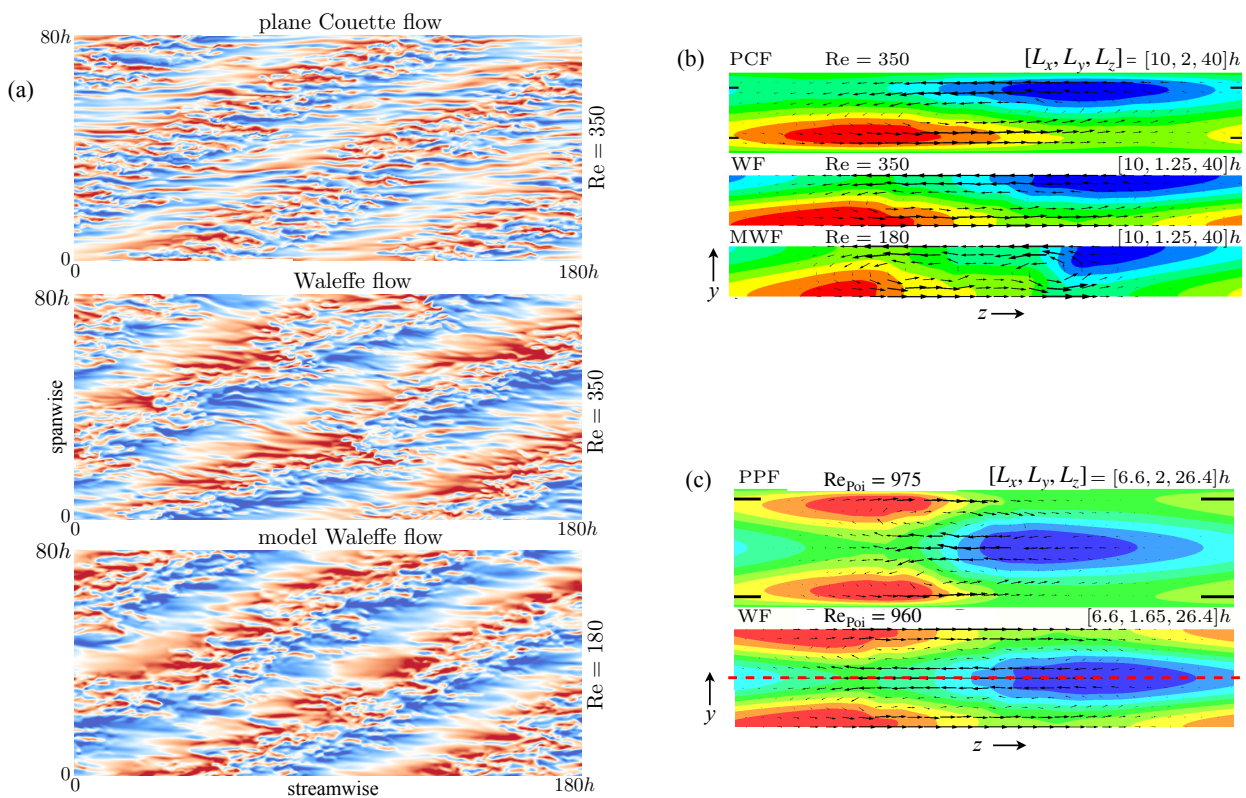

Figure 12

(a) Banded turbulence visualised by instantaneous streamwise velocity at the midplane, with contours from negative (blue) to positive (red) velocity in plane Couette flow, Waleffe flow and model Waleffe flow. (b) Mean flow for turbulent laminar patterns in plane Couette flow (PCF), Waleffe flow (WF), model Waleffe flow (MWF), and (c) their plane Poiseuille equivalents (PPF, $\mathrm{WF})$. Flows are calculated in a tilted domain like that of figure $2(\mathrm{~b})$ and averages are taken in time and in the band-parallel direction. Planes shown are in the band-perpendicular direction $z$ and in the wall-normal direction $y$. Arrows show the flow in these planes, while colors show the magnitude of the flow in the band-parallel direction. The $y$-direction has been stretched by a factor of 3 and black horizontal tick marks in (b) PCF and (c) PPF indicate the bounds of the interior region to which Waleffe flow corresponds. Lengths are in units of the half-gap, $h$, for PCF and PPF. For WF and MWF, $h$ is the half-gap of the corresponding wall-bounded PCF and PPF flows. From Chantry et al. (2016).

\section{Model Waleffe flow: directed percolation}

We have seen that rigid walls are not necessary to reproduce the basic phenomenology of transition to turbulence in plane Couette and Poiseulle flows. This has not only important theoretical consequences, but also practical ones, since this allows the high wall-normal resolution requirements of boundary layers to be avoided. We take a step further and seek a minimal model in the wall-normal direction which reproduces the phenomenology of transitional plane Couette flow. We expand $(u, v, w)$ in low-order trigonometric functions as follows:

$$
\begin{aligned}
& u(x, y, z)=u_{0}(x, z)+u_{1}(x, z) \sin (\beta y)+u_{2}(x, z) \cos (2 \beta y)+u_{3}(x, z) \sin (3 \beta y), \\
& v(x, y, z)=v_{1}(x, z) \cos (\beta y)+v_{2}(x, z) \sin (2 \beta y)+v_{3}(x, z) \cos (3 \beta y), \\
& w(x, y, z)=w_{0}(x, z)+w_{1}(x, z) \sin (\beta y)+w_{2}(x, z) \cos (2 \beta y)+w_{3}(x, z) \sin (3 \beta y),
\end{aligned}
$$


where $\beta=\pi /(2 H)$. In order to insure incompressibility, we use a poloidal-toroidal plus mean-mode representation

$$
\mathbf{u}=f(y) \mathbf{e}_{x}+g(y) \mathbf{e}_{z}+\nabla \times \psi(x, y, z) \mathbf{e}_{\mathbf{y}}+\nabla \times \nabla \times \phi(x, y, z) \mathbf{e}_{\mathbf{y}},
$$

where $f, g$ and $\psi$ match the $y$-formulation of $u$ and $\phi$ matches that of $v$. Because $\psi$ and $\phi$ are taken to be periodic in $x$ and $z$, their derivatives cannot produce functions that are constant in $x$ and $z$, and so the mean modes $f, g$ must be included explicitly to achieve a general, valid representation of $\mathbf{u}$ (e.g., Marqués 1990). Substituting (7) into the NavierStokes equations and applying Fourier orthogonality in $y$, we derive our governing equations, which are seven PDEs in $(x, z, t)$ and six ODEs for the non-constant components of $f$ and $g$. The original eight-ODE model, derived by Waleffe (1997) to illustrate the self-sustaining process, is contained within the system and can be recovered by reducing the number of modes in $y$ and imposing a single Fourier wavenumber in $x$ and $z$. Our model is inspired by a series of models (Manneville \& Locher 2000, Lagha \& Manneville 2007, Seshasayanan \& Manneville 2015, Moehlis et al. 2004) of plane Couette and Waleffe flow.

Not only can the degrees of freedom economized in the wall-normal direction be used to increase the horizontal degrees of freedom, but since the length scales in the horizontal directions tend to mimic those in the wall-normal direction, the elimination of the boundary layers leads to economy in resolution in the horizontal directions as well. In particular, only 4 modes per horizontal spatial unit are needed, compared with 10 for plane Couette flow.

In simulations performed in very large domains, we add a horizontal drag force, sometimes called Rayleigh or Ekman friction:

$$
\mathbf{F}_{\text {Rayl }} \equiv-\sigma\left(u \mathbf{e}_{x}+w \mathbf{e}_{z}\right)
$$

to the Navier-Stokes equations. This force damps all modes, but its effect is most important on modes with no vertical curvature and little horizontal curvature. These would otherwise decay extremely slowly in Waleffe flow and which are absent in Couette flow. The value $\sigma=10^{-2}$ reproduces the damping to which these modes would be subjected in the wall regions of the corresponding Couette flow.

Model Waleffe flow (MWF) displays qualitatively the same transitional phenomena as Waleffe flow and plane Couette flow, but at lower Reynolds numbers. The turbulent-laminar bands shown for MWF in figure 12(a) and (b) occur in the approximate Reynolds number range of $[125,230]$, using the definitions given in equation (5). Inclusion of the drag force (8) shifts upwards the Reynolds number necessary to produce the same phenomena, but these values still remain far below those for plane Couette or Waleffe flow.

Figure 13 shows a simulation at $R e=160$ starting from an initial vortex, following Schumacher \& Eckhardt (2001). A localized turbulent spot develops, with its corresponding large-scale quadrupolar flow, then a spanwise elongated turbulent patch, and finally a complex banded form. This evolution matches that seen in simulations of plane Couette flow by Duguet et al. (2010) and Duguet \& Schlatter (2013). The turbulent spot in the early stage of development was first studied in plane Couette flow by Lundbladh \& Johansson (1991) and Tillmark \& Alfredsson (1992). More recently, the evolution of spots has been investigated as a means to understand the mechanisms of turbulent-laminar interface growth, as well as the development and role of large-scale flows; see Duguet \& Schlatter (2013), Lemoult, Gumowski, Aider \& Wesfreid (2014), Couliou \& Monchaux (2015, 2018).

In the previous sections, we have mentioned lower bounds for the existence of isolated bands, but these have been only approximate. A long-standing and fundamental question 

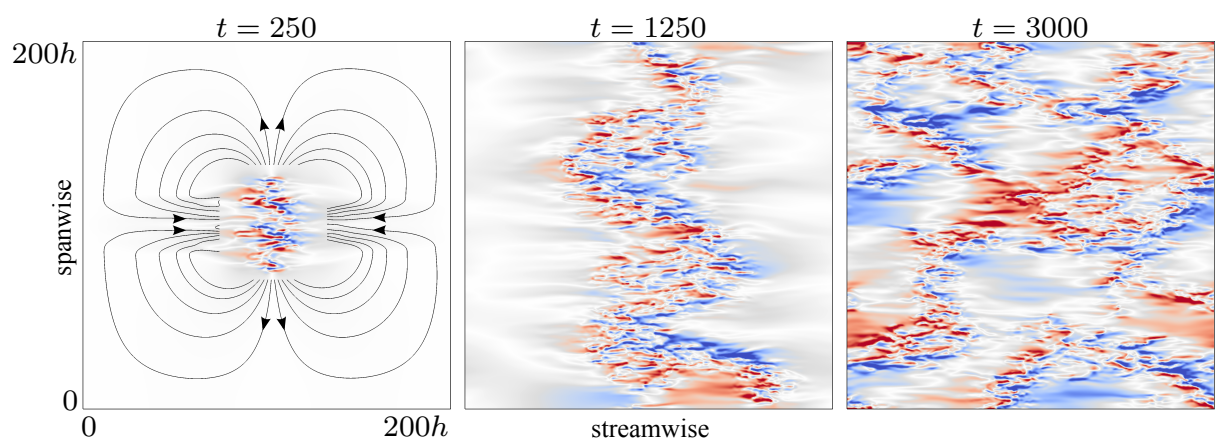

Figure 13

Growth of a turbulent spot in model Waleffe flow. The flow is initialised with a poloidal vortex and subsequent evolution is visualised by streamwise velocity at the midplane. At early times $(t=250)(\mathrm{a})$, a large-scale quadrupolar flow dominates as shown by streamlines of the $y$-averaged flow (contour lines, only plotted away from the spot for visibility). By $t=1250$ bands begin to develop and form a zigzag across the domain (b). The bands continue to grow, and by $t=3000 \mathrm{a}$ complex array of bands fills the domain (c). From Chantry et al. (2016).

has been whether the transition to turbulence is discontinuous or continuous. The CEASaclay group (Daviaud, Hegseth \& Bergé 1992, Bottin, Daviaud, Manneville \& Dauchot 1998, Bottin \& Chaté 1998) has extensively investigated this question for plane Couette flow; Manneville \& Dauchot (2001), Manneville (2015) has stressed that the question must be addressed in the spatiotemporal context.

We have used model Waleffe flow to answer this question (Chantry, Tuckerman \& Barkley 2017) by carrying out simulations in an extremely large domain of size $[2560,1.25,2560]$. Figure 14 shows the instantaneous streamwise velocity at the midplane for such a simulation, at $R e=173.824$ with $\sigma=10^{-2}$, and illustrates precisely why such large domains are necessary for this type of study. The smaller domains used in past experiments and simulations, also shown in figure 14, would be likely to detect no turbulence under these conditions and this is in fact the case. We define the turbulent fraction $F_{t}$ to be the fractional area of the horizontal domain for which the height-integrated energy of the deviation from laminar flow is greater than a threshold value, 0.01, i.e. the colored areas of figure 14. For the large domain of figure 14, a continuous dependence of $F_{t}$ on $R e$ is obtained, with a threshold $R e_{c} \approx 173.80$, as shown in figure 15(a). For simulations of MWF carried out in a smaller domain of size [380,1.25,70], figure $15(\mathrm{~b})$ shows that $F_{t}$ behaves discontinuously with Re. Philip \& Manneville (2011) computed a discontinuous transition to turbulence in plane Couette flow whose Reynolds-number threshold decreased with increasing domain size, but all of their domains were considerably smaller than any of those depicted in figure 14. The transition to turbulence in MWF is continuous, but only in the limit of infinite domain size; even in figure 15(a), there exists a minimum non-zero value of $F_{t}$ based on the domain size. In the classic hydrodynamic pattern-forming systems such as co-rotating Taylor-Couette flow or Rayleigh-Bénard convection, the transition is to a new state which exists everywhere, but has infinitesimally small amplitude. In contrast, in MWF (and presumably also in plane Couette flow and Poiseuille flow), transition to turbulence occurs via the increasing density occupied by the turbulent state in an otherwise 


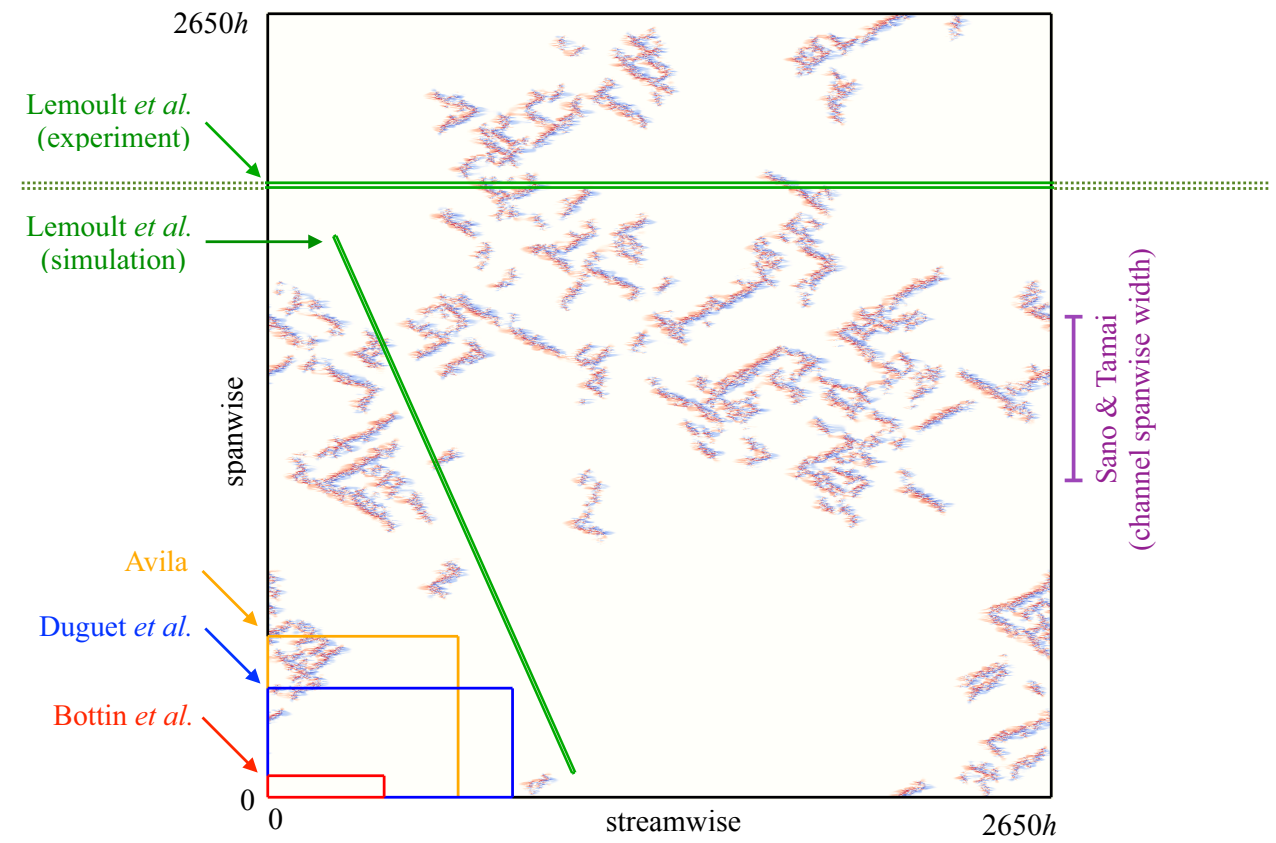

Figure 14

Instantaneous depiction of intermittent turbulence just above onset of sustained turbulence. Streamwise velocity in the midplane is shown for a simulation of model Waleffe flow in a domain with dimensions $[2560,1.25,2560]$ at reduced Reynolds number $\epsilon=1.4 \times 10^{-4}$ with Rayleigh friction factor $\sigma=10^{-2}$ after $1.2 \times 10^{6}$ time units. Laminar flow is seen as white. The turbulence fraction is $F_{t} \approx 0.1$. Experimental and numerical domains of Bottin et al. (1998), Duguet et al. (2010), Avila (2013) and are overlaid in red, blue and orange respectively. Part of the quasi-one-dimensional domain of Lemoult et al. (2016) is shown in green and the spanwise width of the plane Poiseuille experiment of Sano \& Tamai (2016) is indicated in purple on the right. From Chantry et al. (2017).

laminar background, not via its increasing amplitude in any given area or volume.

Our simulations of MWF demonstrate a more specific aspect of turbulent transition. Noting that in subcritical shear flows, turbulence could spread into laminar regions but cannot arise spontaneously, Pomeau (1986) postulated that this transition might belong to the universality class of directed percolation, an idea supported by Manneville \& Dauchot (2001). This would imply not only that $F_{t}$ varies continuously with Reynolds number, but also that power laws with specific predicted exponents would hold near onset. Figure 15(a) shows that $F_{t} \sim\left(R e-R e_{c}\right)^{\beta}$, where $\beta$ has the value 0.583 predicted for directed percolation with two extended directions (Lübeck 2004). Figure 16 shows the temporal evolution of $F_{t}$ for various Reynolds numbers near $R e_{c}$. Above $R e_{c}, F_{t}$ eventually saturates at the finite values plotted in figure $15(\mathrm{a})$, while below $R e_{c}, F_{t}$ eventually decreases to zero. The supplemental movie shows the evolution of MWF in our large domain, for cases with $R e \approx R e_{c}$ and $R e>R e_{c}$. The theory of directed percolation makes quantitative predictions about this behavior (Lübeck 2004). When time and the turbulent fraction are rescaled as shown in $16(\mathrm{~b})$, to $t|\epsilon|^{\nu \|}$ and $t^{\alpha} F_{t}$, with the definition $\epsilon \equiv\left(R e-R e_{c}\right) / R e_{c}$ and 

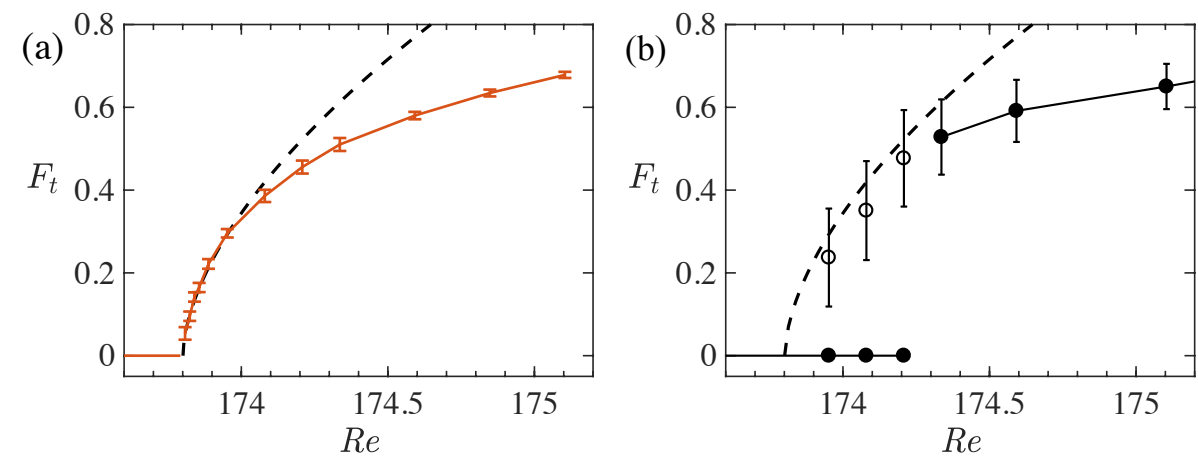

Figure 15

Bifurcation diagrams for the transition to turbulence. (a) Continuous transition in domain of size $[2560,1.25,2560]$. Equilibrium turbulence fraction $F_{t}$ is plotted as a function of Re. Points and error bars denote mean and standard deviation of $F_{t}$. (b) Discontinuous transition in a domain of size $[380,1.25,70]$, approximately that of the experiments by Bottin \& Chaté (1998). Filled points denote sustained turbulence, while open points denote the turbulence fraction of long-lived transient turbulence. Black dashed curves show the directed percolation power law from the large domain. Adapted from Chantry et al. (2017).
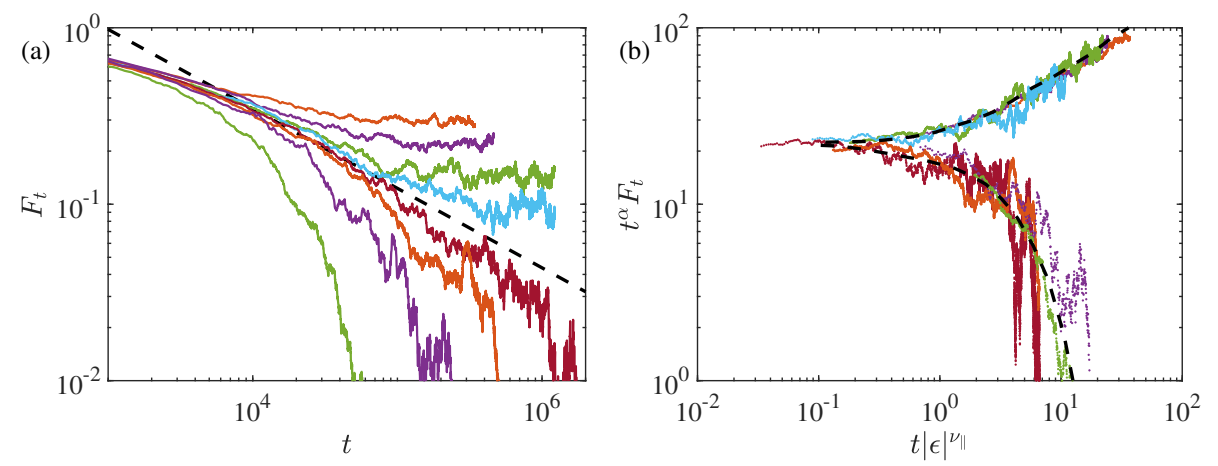

Figure 16

(a) Turbulence fraction as function of time for a range of Reynolds numbers initialised with uniform turbulence. Above criticality, the turbulence fraction saturates at a finite value, and below it falls to zero. At criticality, the turbulence fraction decays in time as a power law $F_{t} \sim t^{-\alpha}$ with the directed percolation exponent $\alpha \simeq 0.4505$ (dashed line). Colored lines from highest to lowest $F_{t}$ correspond to evolution at reduced Reynolds numbers ranging from $\epsilon=0.87 \times 10^{-3}$ to $\epsilon=-1.33 \times 10^{-3}$. (b) Data above and below criticality collapse onto two scalings (black dashed curves) when the directed percolation exponents are used to rescale time and turbulence fraction. From Chantry et al. (2017).

the exponents have the theoretical values $\alpha \simeq 0.4505$ and $\nu_{\|} \simeq 1.295$, the data collapse on two curves, one for above-critical evolution and the other for below-critical evolution.

Counter-rotating Taylor-Couette flow with a very narrow gap, and hence minimal curvature, has been used as a way of approaching plane Couette flow. Shi, Avila \& Hof (2013) carried out simulations of Taylor-Couette flow in a long tilted domain like that of figure 2 in order to determine the typical statistical lifetimes as a function of Reynolds number for decay and splitting, as in figure 6(a) and (c). The two curves cross at $R e=325$ (using the 

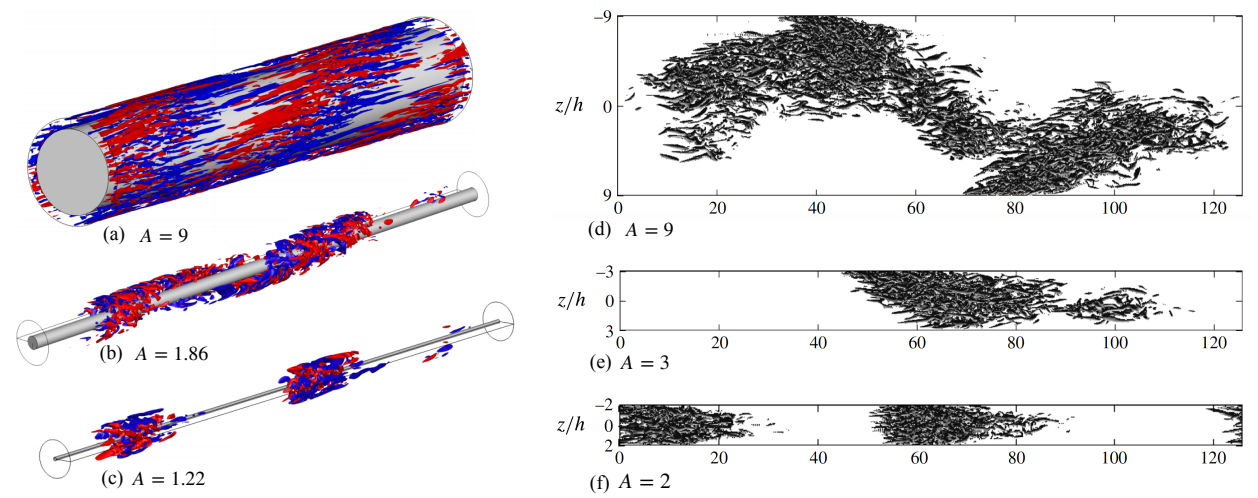

Figure 17

Turbulent-laminar patterns in annular pipe flow $(a, b, c)$ adapted from Ishida et al. (2016), and in duct flow (d,e,f) adapted from Takeishi et al. (2015). The aspect ratio $A$ is defined as

$A=\left(r_{\text {out }}+r_{\text {in }}\right) /\left(r_{\text {out }}-r_{\text {in }}\right)$ for annular pipe flow and as $A=L_{\text {span }} /$ height for duct flow. For large $A$, there are two extended directions and turbulence takes the form of oblique bands. For small $A$, only the streamwise direction is extended and turbulence takes the form of puffs.

Lengths in (d-f) are in units of the half-height, $h$.

conventions of plane Couette flow), which defines a critical point beyond which splitting dominates decay, as pioneered in Avila, Moxey, de Lozar, Avila, Barkley \& Hof (2011) for pipe flow. This value should approximate the directed percolation threshold, were such a calculation to be carried out for fully resolved wall-bounded plane Couette flow. Note that this implies that the states with $R e \leq 325$ seen in figure 6 are probably long transients. The value of $R e=323$ was already mentioned in Bottin et al. (1998), although in reference to discontinuous experimental results like those of figure 16(b). Lemoult, Shi, Avila, Jalikop, Avila \& Hof (2016) combined the narrow-gap limit with a drastic reduction in the spanwise direction by reducing the axial height (see figure 14), so that turbulence would take the form of patches rather than bands. They showed that the transition to turbulence for this case verified the scalings of one-dimensional directed percolation.

An important open question is the nature of the transition in plane Poiseuille flow. Sano \& Tamai (2016) report evidence for directed percolation with a critical Reynolds number of $R e_{\text {Poi }}=830$. However, as seen in figure 9 (b) of section 3 , this transition is preceded by the formation of robust oblique turbulent bands at Reynolds numbers as low as $R e_{\text {Poi }}=660$ (Xiong et al. 2015, Tao et al. 2018). Thus the transition in plane Poiseuille flow may be more complicated than standard directed percolation.

\section{Other flows}

Oblique turbulent bands form when there is one confined and two extended directions. In addition to the previously mentioned study by Lemoult et al. (2016), several other scenarios have been explored in which the bands become patches or puffs when one of the extended directions is reduced. Figure 17 shows pressure-driven flows in two parameterized geometries: axially driven flow between two concentric cylinders called annular pipe flow (Ishida, Duguet \& Tsukahara 2016), and flow through a rectangular duct (Takeishi, Kawahara, Wak- 

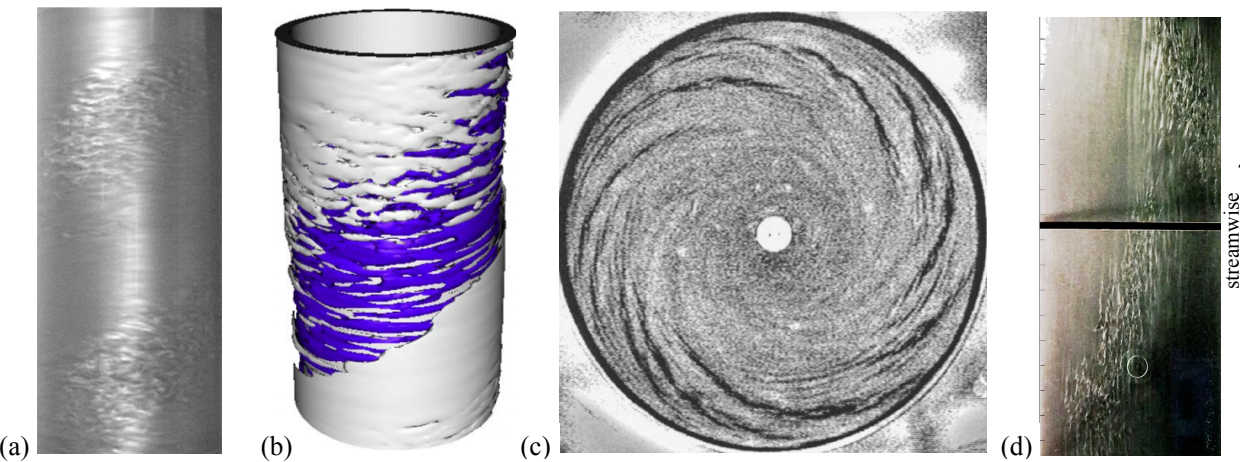

Figure 18

Turbulent bands in counter-rotating Taylor-Couette (a) experiment of Goharzadeh \& Mutabazi (2001) and (b) simulation of Meseguer et al. (2009). (c) Spiraling turbulent bands in experiment of Cros \& Le Gal (2002) of torsional Couette flow between closely spaced differentially rotating disks. (d) Oblique turbulent patch in Poiseuille-Couette flow from Klotz et al. (2017).

abayashi, Uhlmann \& Pinelli 2015). The analogue of the spanwise direction (perpendicular to the gap and to the streamwise direction) is the circumference for the annular pipe and the larger cross-sectional dimension for the duct. For both flows, a spanwise-to-gap aspect ratio can be defined; both approach plane Poiseuille flow in the limit of infinite aspect ratio and resemble pipe flow in the limit of small aspect ratio. Figure 17 shows oblique bands for large $A$ and localized puffs for small $A$.

For counter-rotating Taylor-Couette flow, in which turbulent bands were first discovered, the directions analogous to streamwise and spanwise are azimuthal and axial. An experimental realization by Goharzadeh \& Mutabazi (2001) is shown in figure 18, along with a numerical realization by Meseguer, Mellibovsky, Avila \& Marques (2009); see also Dong (2009). Rotating plane Couette flow (where the axis of rotation is oriented in the spanwise direction and located at mid-gap) is closely related to Taylor-Couette flow; this flow has been surveyed and turbulent bands observed by Tsukahara, Tillmark \& Alfredsson (2010) and Brethouwer et al. (2012). Torsional Couette flow, the flow between two differentially rotating closely spaced disks, is another variant, in which the analogue of the streamwise direction is azimuthal, as in Taylor-Couette flow, but the analogues of the spanwise and gap directions are radial and axial. Figure 18(c) shows an experimental realization of spiralling widely spaced turbulent bands in this flow by Cros \& Le Gal (2002). An oblique turbulent patch has also been seen in a Poiseuille-Couette experiment with one moving wall and zero mean flux by Klotz, Lemoult, Frontczak, Tuckerman \& Wesfreid (2017), as shown in figure $18(\mathrm{~d})$.

Turbulent bands have been sought in other flows, with a view to determining which features favor or suppress them. Brethouwer et al. (2012) observed bands in Couette and Poiseuille flows subjected to stabilizing influences such as stratification, cyclonic rotation, or a magnetic field, but at higher and wider Reynolds-number ranges. Spatially localized turbulence was also studied by Zikanov, Krasnov, Boeck, Thess \& Rossi (2014) and by Deusebio, Caulfield \& Taylor (2015) in the presence of a magnetic field and stratification, respectively. Wang, Shekar \& Graham (2017) observed oblique bands in simulations of viscoelastic turbulence in channel flow. Ishida, Brethouwer, Duguet \& Tsukahara (2017) 
found that rough walls tend to suppress turbulent bands in plane Poiseuille flow. Khapko, Schlatter, Duguet \& Henningson (2016) found that the asymptotic suction boundary layer does not support oblique turbulent bands. They provided evidence that this is due to the lack of vertical confinement, more specifically the fact that a boundary layer, bordered by only one wall, allows large-scale wall-normal flows. This is consistent with the analysis of Duguet \& Schlatter (2013) on the role of incompressibility of the large-scale flows in generating oblique structures.

\section{Conclusion}

In all of the transitional flows we have depicted, turbulence takes the form of long and well-separated oblique bands. These bands exist whenever the domain is sufficiently large to accomodate them, and this is true even very close to the threshold for turbulence, e.g. in figures $6(\mathrm{~d})$ or 14 . Their widths and angles remain comparable to those in a regularly spaced pattern, even when the bands are isolated or sparsely scattered throughout the domain. They occur in the hydrodynamic flows discussed in detail here, i.e. plane Couette flow and Poiseuille flow, and also in many other flows such as Taylor-Couette flow, torsional Couette flow, and annular pipe flow.

A tilted rectangular domain with periodic boundary conditions (MBU) provides the minimal horizontal domain in which one or a few turbulent bands can be computed. By reproducing their phenomenology with free-slip boundary conditions, we have shown that the boundary layers are unimportant and the effect of the walls is only to produce shear and confinement. In order to carry out simulations in domains containing a large number of bands, we have shown that a minimal model of the vertical dependence retains the qualitative properties of transitional plane Couette flow and we have used this model to show that the transition to turbulence via band extinction is in the universality class of directed percolation.

Turbulent bands assemble and organize the much smaller streamwise streaks and vortices of which they are composed. First considered as an exotic manifestation and generalization of pattern formation, turbulent bands have turned out to be elementary and fundamental components of transitional flows, much like the streaks and vortices. They oc-

cupy an intermediate position in the hierarchy between the large horizontal dimensions of a domain and the vertical gap size which is the scale of the streaks and vortices. The bands exist symbiotically with large-scale flows; the interaction between the two is responsible for maintaining them. Yet, despite the considerable interest and effort devoted to their study, the mechanisms producing the bands remain, at best, incompletely understood. Their angles and widths cannot yet be quantitatively explained by a predictive theory from first principles.

\section{ACKNOWLEDGMENTS}

This work was performed using high performance computing resources provided by the Institut du Developpement et des Ressources en Informatique Scientifique (IDRIS) of the Centre National de la Recherche Scientifique (CNRS), coordinated by GENCI (Grand Equipement National de Calcul Intensif) through a series of grants, including 1119, i20070211119, i20080211119, i2009021119, i2010021119, i2011021119, i2012021119, i20132a1119, i20142a1119, i20152a1119 i20162a1119 and A0022A01119. The authors are 
grateful for support from the Agence Nationale de la Recherche (ANR) through the TRANSFLOW project. This research was supported in part by the National Science Foundation under Grant No. NSF PHY-1748958 and by a CNRS-Royal Society grant.

\section{LITERATURE CITED}

Andereck CD, Liu S, Swinney HL. 1986. Flow regimes in a circular Couette system with independently rotating cylinders. J. Fluid Mech. 164:155-183

Avila K. 2013. Shear flow experiments: Characterizing the onset of turbulence as a phase transition, $\mathrm{PhD}$ thesis, Georg-August University School of Science

Avila K, Moxey D, de Lozar A, Avila M, Barkley D, Hof B. 2011. The onset of turbulence in pipe flow. Science 333:192-196

Barkley D, Tuckerman LS. 2005a. Computational study of turbulent laminar patterns in Couette flow. Phys. Rev. Lett. 94:014502

Barkley D, Tuckerman LS. 2005b. Turbulent-laminar patterns in plane Couette flow, In IUTAM Symposium on Laminar-Turbulent Transition and Finite Amplitude Solutions, eds. T Mullin, R Kerswell. Springer

Barkley D, Tuckerman LS. 2007. Mean flow of turbulent-laminar patterns in plane Couette flow. J. Fluid Mech. 576:109-137

Bottin S, Chaté H. 1998. Statistical analysis of the transition to turbulence in plane Couette flow. Eur. Phys. J. B 6:143-155

Bottin S, Daviaud F, Manneville P, Dauchot O. 1998. Discontinuous transition to spatiotemporal intermittency in plane Couette flow. Europhys. Lett. 43:171-176

Brethouwer G, Duguet Y, Schlatter P. 2012. Turbulent-laminar coexistence in wall flows with Coriolis, buoyancy or Lorentz forces. J. Fluid Mech. 704:137-172

Campbell D, Farmer D, Crutchfield J, Jen E. 1985. Experimental mathematics: the role of computation in nonlinear science. Commun. ACM 28:374-384

Chantry M, Tuckerman LS, Barkley D. 2016. Turbulent-laminar patterns in shear flows without walls. J. Fluid Mech. 791:R8

Chantry M, Tuckerman LS, Barkley D. 2017. Universal continuous transition to turbulence in a planar shear flow. J. Fluid Mech. 824:R1

Coles D, van Atta C. 1966. Progress report on a digital experiment in spiral turbulence. AIAA Journal 4:1969-1971

Couliou M, Monchaux R. 2015. Large-scale flows in transitional plane Couette flow: a key ingredient of the spot growth mechanism. Phys. Rev. Fluids 27:034101

Couliou M, Monchaux R. 2018. Childhood of turbulent spots in a shear flow. Phys. Rev. Fluids 3:123901

Cros A, Le Gal P. 2002. Spatiotemporal intermittency in the torsional Couette flow between a rotating and a stationary disk. Phys. Fluids 14:3755-3765

Cvitanović P, Eckhardt B. 1989. Periodic-orbit quantization of chaotic systems. Phys. Rev. Lett. 63:823

Daviaud F, Hegseth J, Bergé P. 1992. Subcritical transition to turbulence in plane Couette flow. Phys. Rev. Lett. 69:2511

Deguchi K, Hall P. 2015. Asymptotic descriptions of oblique coherent structures in shear flows. J. Fluid Mech. 782:356-367

Deusebio E, Caulfield C, Taylor J. 2015. The intermittency boundary in stratified plane Couette flow. J. Fluid Mech. 781:298-329

Dong S. 2009. Evidence for internal structures of spiral turbulence. Phys. Rev. E 80:067301

Duguet Y, Schlatter P. 2013. Oblique laminar-turbulent interfaces in plane shear flows. Phys. Rev. Lett. 110:034502

Duguet Y, Schlatter P, Henningson DS. 2010. Formation of turbulent patterns near the onset of 
transition in plane Couette flow. J. Fluid Mech. 650:119-129

Feynman RP. 1964. Lecture Notes in Physics. Reading: Addison-Wesley

Fukudome K, Iida O. 2012. Large-scale flow structure in turbulent Poiseuille flows at low-Reynolds numbers. J. Fluid Sci. Tech. 7:181-195

Gibson JF, Halcrow J, Cvitanović P. 2009. Equilibrium and travelling-wave solutions of plane Couette flow. J. Fluid Mech. 638:243-266

Gibson JF, Reetz F, Azimi S, Ferraro A, Kreilos T, et al. 2019. Channelflow 2.0. in preparation See channelflow.ch

Goharzadeh A, Mutabazi I. 2001. Experimental characterization of intermittency regimes in the Couette-Taylor system. Eur. Phys. J. B 19:157-162

Goharzadeh A, Mutabazi I. 2008. The phase dynamics of spiral turbulence in the Couette-Taylor system. Eur. Phys. J. B 66:81-84

Hamilton JM, Kim J, Waleffe F. 1995. Regeneration mechanisms of near-wall turbulence structures. J. Fluid Mech. 287:317-348

Hashimoto S, Hasobe A, Tsukahara T, Kawaguchi Y, Kawamura H. 2009. An experimental study on turbulent-stripe structure in transitional channel flow, In Turbulence, Heat and Mass Transfer 6, eds. YN K. Hanjalić, S Jakirlić. ArXiv:1406.1378 [physics.flu-dyn]

Hegseth JJ, Andereck CD, Hayot F, Pomeau Y. 1989. Spiral turbulence and phase dynamics. Phys. Rev. Lett. 62:257

Henderson RD, Karniadakis GE. 1995. Unstructured spectral element methods for simulation of turbulent flows. J. Comput. Phys. 122:191-217

Ishida T, Brethouwer G, Duguet Y, Tsukahara T. 2017. Laminar-turbulent patterns with rough walls. Phys. Rev. Fluids 2:073901

Ishida T, Duguet Y, Tsukahara T. 2016. Transitional structures in annular Poiseuille flow depending on radius ratio. J. Fluid Mech. 794

Kanazawa T. 2018. Lifetime and growing process of localized turbulence in plane channel flow, $\mathrm{PhD}$ thesis, Osaka University

Kawahara G, Kida S. 2001. Periodic motion embedded in plane Couette turbulence: regeneration cycle and burst. J. Fluid Mech. 449:291-300

Kawahara G, Uhlmann M, Van Veen L. 2012. The significance of simple invariant solutions in turbulent flows. Annu. Rev. Fluid Mech. 44:203-225

Khapko T, Schlatter P, Duguet Y, Henningson D. 2016. Turbulence collapse in a suction boundary layer. J. Fluid Mech. 795:356-379

Klotz L, Lemoult G, Frontczak I, Tuckerman LS, Wesfreid JE. 2017. Couette-Poiseuille flow experiment with zero mean advection velocity: Subcritical transition to turbulence. Phys. Rev. Fluids 2:043904

Kushwaha A, Park JS, Graham MD. 2017. Temporal and spatial intermittencies within channel flow turbulence near transition. Phys. Rev. Fluids 2:024603

Lagha M, Manneville P. 2007. Modeling of plane Couette flow. i. large scale flow around turbulent spots. Phys. Fluids 19:094105

Lemoult G, Gumowski K, Aider JL, Wesfreid JE. 2014. Turbulent spots in channel flow: an experimental study. Eur. Phys. J. E 37:25

Lemoult G, Shi L, Avila K, Jalikop SV, Avila M, Hof B. 2016. Directed percolation phase transition to sustained turbulence in Couette flow. Nat. Phys. 12:254-258

Lübeck S. 2004. Universal scaling behavior of non-equilibrium phase transitions. Int. J. Mod. Phys. B 18:3977-4118

Lundbladh A, Johansson AV. 1991. Direct simulation of turbulent spots in plane Couette flow. J. Fluid Mech. 229:499-516

Manneville P. 2015. On the transition to turbulence of wall-bounded flows in general, and plane Couette flow in particular. Eur. J. Mech. B-Fluid 49:345-362

Manneville P, Dauchot O. 2001. Patterning and transition to turbulence in subcritical systems: 
the case of plane Couette flow, In Sitges. Conf. on coherent structures in classical systems, eds. D Reguera, L Bonilla, J Rubi. Springer

Manneville P, Locher F. 2000. A model for transitional plane Couette flow. C.R. Acad. Sci. Paris, Serie IIb 328:159-164

Marqués F. 1990. On boundary conditions for velocity potentials in confined flows: Application to Couette flow. Phys. Fluids A 2:729-737

Meseguer A, Mellibovsky F, Avila M, Marques F. 2009. Instability mechanisms and transition scenarios of spiral turbulence in Taylor-Couette flow. Phys. Rev. E 80:046315

Mizuno Y, Jiménez J. 2013. Wall turbulence without walls. J. Fluid Mech. 723:429-455

Moehlis J, Faisst H, Eckhardt B. 2004. A low-dimensional model for turbulent shear flows. New J. Phys. 6:56

Nagata M. 1990. Three-dimensional finite-amplitude solutions in plane Couette flow: bifurcation from infinity. J. Fluid Mech. 217:519-527

Philip J, Manneville P. 2011. From temporal to spatiotemporal dynamics in transitional plane Couette flow. Phys. Rev. E 83:036308

Podvin B, Fraigneau Y. 2011. Synthetic wall boundary conditions for the direct numerical simulation of wall-bounded turbulence. J. Turbul. 12:1-26

Pomeau Y. 1986. Front motion, metastability and subcritical bifurcations in hydrodynamics. Physica D 23:3-11

Prigent A, Grégoire G, Chaté H, Dauchot O. 2003. Long-wavelength modulation of turbulent shear flows. Physica D 174:100-113

Prigent A, Grégoire G, Chaté H, Dauchot O, van Saarloos W. 2002. Large-scale finite-wavelength modulation within turbulent shear flows. Phys. Rev. Lett. 89:014501

Reetz F, Kreilos T, Schneider TM. 2019. Exact invariant solution reveals the origin of self-organized oblique turbulent-laminar stripes. Nature Comm. 10:2277

Sano M, Tamai K. 2016. A universal transition to turbulence in channel flow. Nat. Phys. 12:249-253

Schneider TM, Marinc D, Eckhardt B. 2010. Localized edge states nucleate turbulence in extended plane Couette cells. J. Fluid Mech. 646:441-451

Schumacher J, Eckhardt B. 2001. Evolution of turbulent spots in a parallel shear flow. Phys. Rev. E 63:046307

Seshasayanan K, Manneville P. 2015. Laminar-turbulent patterning in wall-bounded shear flows: a Galerkin model. Fluid Dyn. Res. 47:035512

Shi L, Avila M, Hof B. 2013. Scale invariance at the onset of turbulence in Couette flow. Phys. Rev. Lett. 110:204502

Takeishi K, Kawahara G, Wakabayashi H, Uhlmann M, Pinelli A. 2015. Localized turbulence structures in transitional rectangular-duct flow. J. Fluid Mech. 782:368-379

Tao J, Eckhardt B, Xiong X. 2018. Extended localized structures and the onset of turbulence in channel flow. Phys. Rev. Fluids 3:011902

Tillmark N, Alfredsson PH. 1992. Experiments on transition in plane Couette flow. J. Fluid Mech. 235:89-102

Tsukahara T, Iwamoto K, Kawamura H, Takeda T. 2006. DNS of heat transfer in a transitional channel flow accompanied by a turbulent puff-like structure, In Turbulence, Heat and Mass Transfer 5, eds. YN K. Hanjalić, S Jakirlić. ArXiv:1406.0586 [physics.flu-dyn]

Tsukahara T, Seki Y, Kawamura H, Tochio D. 2005. DNS of turbulent channel flow at very low Reynolds numbers, In Proc. 4th Int. Symp. on Turbulence and Shear Flow Phenomena. ArXiv:1406.0248 [physics.flu-dyn]

Tsukahara T, Tillmark N, Alfredsson P. 2010. Flow regimes in a plane Couette flow with system rotation. J. Fluid Mech. 648:5-33

Tuckerman LS, Barkley D. 2011. Patterns and dynamics in transitional plane Couette flow. Phys. Fluids 23:041301

Tuckerman LS, Kreilos T, Schrobsdorff H, Schneider TM, Gibson JF. 2014. Turbulent-laminar 
patterns in plane Poiseuille flow. Phys. Fluids 26:114103

Waleffe F. 1997. On a self-sustaining process in shear flows. Phys. Fluids 9:883-890

Wang SN, Shekar A, Graham MD. 2017. Spatiotemporal dynamics of viscoelastic turbulence in transitional channel flow. J. Nonnewton. Fluid Mech. 244:104-122

Xiong X, Tao J, Chen S, Brandt L. 2015. Turbulent bands in plane-Poiseuille flow at moderate Reynolds numbers. Phys. Fluids 27:041702

Zikanov O, Krasnov D, Boeck T, Thess A, Rossi M. 2014. Laminar-turbulent transition in magnetohydrodynamic duct, pipe, and channel flows. Appl. Mech. Rev. 66:030802 